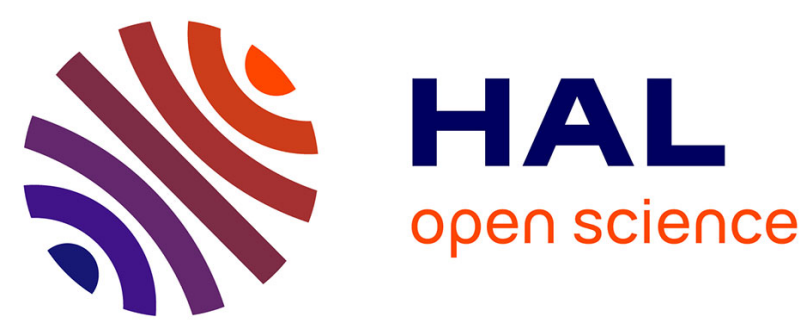

\title{
Insertion and activation of functional Bacteriorhodopsin in a floating bilayer
}

Tetiana Mukhina, Yuri Gerelli, Arnaud Hemmerle, Alexandros Koutsioubas, Kirill Kovalev, Jean-Marie Teulon, Jean-Luc Pellequer, Jean Daillant, Thierry Charitat, Giovanna Fragneto

\section{- To cite this version:}

Tetiana Mukhina, Yuri Gerelli, Arnaud Hemmerle, Alexandros Koutsioubas, Kirill Kovalev, et al.. Insertion and activation of functional Bacteriorhodopsin in a floating bilayer. Journal of Colloid and Interface Science, 2021, 597, pp.370-382. 10.1016/j.jcis.2021.03.155 hal-03216526

\section{HAL Id: hal-03216526 \\ https://hal.univ-grenoble-alpes.fr/hal-03216526}

Submitted on 4 May 2021

HAL is a multi-disciplinary open access archive for the deposit and dissemination of scientific research documents, whether they are published or not. The documents may come from teaching and research institutions in France or abroad, or from public or private research centers.
L'archive ouverte pluridisciplinaire HAL, est destinée au dépôt et à la diffusion de documents scientifiques de niveau recherche, publiés ou non, émanant des établissements d'enseignement et de recherche français ou étrangers, des laboratoires publics ou privés. 
Regular Article

\title{
Insertion and activation of functional Bacteriorhodopsin in a floating bilayer
}

\author{
Tetiana Mukhina ${ }^{\mathrm{a}, \mathrm{b}, 1}$, Yuri Gerelli ${ }^{\mathrm{a}, \mathrm{c}, *}$, Arnaud Hemmerle ${ }^{\mathrm{d}}$, Alexandros Koutsioubas ${ }^{\mathrm{e}}$, Kirill Kovalev ${ }^{\mathrm{f}, \mathrm{g}, \mathrm{e}, \mathrm{h}, \mathrm{i}, \mathrm{j}}$, \\ Jean-Marie Teulon ${ }^{\mathrm{f}}$, Jean-Luc Pellequer ${ }^{\mathrm{f}}$, Jean Daillant ${ }^{\mathrm{d}}$, Thierry Charitat ${ }^{\mathrm{b}, *}$, Giovanna Fragneto ${ }^{\mathrm{a}, *}$ \\ a Institut Laue-Langevin, 71 av.des Martyrs, BP 156, 38042 Grenoble Cedex, France \\ ${ }^{\mathrm{b}}$ Institut Charles Sadron, Université de Strasbourg, CNRS, UPR 22, 67034 Strasbourg, France \\ ${ }^{\mathrm{c}}$ Marche Polytechnic University, Department of Life and Environmental Sciences, Via Brecce Bianche, 60131 Ancona, Italy \\ ' Synchrotron SOLEIL, L'Orme des Merisiers, Saint-Aubin, BP 48, F-91192 Gif-sur-Yvette Cedex, France \\ e Jülich Centre for Neutron Science (JCNS) at Heinz Maier-Leibnitz Zentrum (MLZ), Forschungszentrum Jülich GmbH, Lichtenbergstr. 1, 85748 Garching, Germany \\ ${ }^{\mathrm{f}}$ Univ. Grenoble Alpes, CEA, CNRS, Institut de Biologie Structurale (IBS), F-38000 Grenoble, France \\ ${ }^{\mathrm{g}}$ Institute of Biological Information Processing (IBI-7), Structural Biochemistry, Forschungszentrum Jülich, 52428, Wilhelm-Johnen-Straße, Jülich, Germany \\ h Jülich Centre for Structural Biology, Forschungszentrum Jülich, 52428, Wilhelm-Johnen-Straße, Jülich, Germany \\ ${ }^{i}$ Research Center for Molecular Mechanisms of Aging and Age-Related Diseases, Moscow Institute of Physics and Technology, 141071, 9 Institutskiy per., Dolgoprudny, Russia \\ ${ }^{\mathrm{j}}$ Institute of Crystallography, RWTH Aachen University, 52066, Jägerstraße 17-19, Aachen, Germany
}

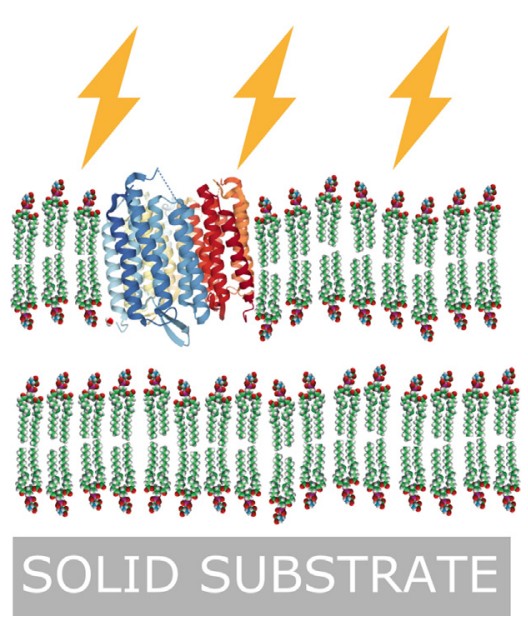

A R T I C L E I N F O

\section{Article history:}

Received 24 November 2020

Revised 26 March 2021

Accepted 27 March 2021

Available online 31 March 2021

\begin{abstract}
A B S T R A C T
The proton pump transmembrane protein bacteriorhodopsin was successfully incorporated into planar floating lipid bilayers in gel and fluid phases, by applying a detergent-mediated incorporation method. The method was optimized on single supported bilayers by using quartz crystal microbalance, atomic force and fluorescence microscopy techniques. Neutron and X-ray reflectometry were used on both single and floating bilayers with the aim of determining the structure and composition of this membrane-
\end{abstract}

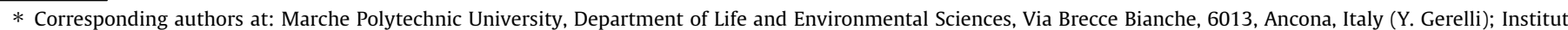

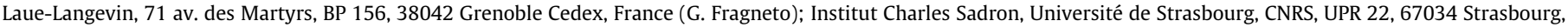
France (T. Charitat).

1 Present address: Technischen Universität Darmstadt, Institut für Festkörperphysik, Hochschulstraße 8, 64289, Darmstadt, Germany
} 
Keywords:

Membrane

Bilayer

Phospholipid

Protein reconstitution

Bacteriorhodopsin

Neutron reflectometry

$\mathrm{X}$-ray reflectometry protein system before and after protein reconstitution at sub-nanometer resolution. Lipid bilayer integrity and protein activity were preserved upon the reconstitution process. Reversible structural modifications of the membrane, induced by the bacteriorhodopsin functional activity triggered by visible light, were observed and characterized at the nanoscale.

(c) 2021 Elsevier Inc. All rights reserved.

\section{Introduction}

${ }^{2}$ Supported lipid bilayers are often used as model membrane systems immobilized onto solid substrates and are capable of interacting with large molecules or molecular assemblies such as vesicles [1], nanoparticles [2], proteins, peptides and biopolymers [3]. Beyond their applications in biotechnology [4], they are important for many fundamental studies in physics (diffusion, mechanical properties, etc.) and biology (trafficking, endocytosis, exocytosis, membrane reshaping, etc.) [5-9] . In fact, by being an almost flat and immobilized system, they allow studies with high resolution techniques such as atomic force microscopy (AFM) [10], fluorescence resonance energy transfer (FRET) [11] and scattering of X-rays or neutrons [12]. One of the main drawbacks of adsorbed bilayers is that, due to their strong interaction with the solid substrate, they do not accurately replicate the conditions of natural membranes, which are inherently fluctuating systems and may enhance the activity of biomolecules such as membrane proteins. For this reason, polymer cushions have been used in the past as spacers between the substrate and the model membrane $[13,14]$. Polymer cushions often induce significant roughness to the supported bilayer, which is not desirable for structural determinations. A more appropriate system for fine structural characterisation, developed by our team in recent decades, has involved the use of an adsorbed lipid bilayer acting as a spacer between a floating bilayer and the substrate [15]. This construct is often referred to as a double bilayer. A floating bilayer is more hydrated and free to fluctuate than an adsorbed bilayer $[16,17]$ and is particularly well-suited to investigate modifications in the bilayer physical properties induced by external stimuli (such as an electric field [18]), as well as bilayer interaction with nanoobjects $[19,20]$ by means of surface scattering techniques. Double bilayers, as well as polymer cushioned lipid bilayers [21], are also promising candidates for the reconstitution of proteins in systems close to their natural environment, i.e. in conditions preserving the original protein activity [10]. The reconstitution process is not trivial. One of the challenges is related to the fragility of the floating layers that are weakly bound to the surface. Nevertheless, the insertion of active transmembrane proteins in floating bilayers opens up prominent perspectives for the study of fundamental biological processes at the molecular level [22], as well as the investigation into fascinating physical properties such as the fluctuations of an active membrane subjected to active athermal noise [23,24].

In this work we aimed to develop a robust protocol for incorporation of bacteriorhodopsin (BR) while maintaining the structural integrity of the bilayers and the functional activity of the protein. Bacteriorhodopsin is a relatively small $(27 \mathrm{kDa})$ and highly

\footnotetext{
${ }^{2}$ AFM = Atomic Force Microscopy; FM = Fluorescence Microscopy; NR = Neutron Reflectometry; XRR = X-ray Reflectometry; LB = Langmuir-Blodgett; LS = LangmuirSchaefer; SLD = neutron scattering length density; $E D=$ electron density; DDM = ndodecyl- $\beta$-D-maltopyranoside; PBS = Phosphate-buffered saline; DSPC = 1,2-distear oyl-sn-glycero-3-phosphocholine; $\mathrm{DPPC}=1,2$-dipalmitoyl-sn-glycero-3phosphocholine; $\mathrm{POPC}=1$-palmitoyl-2-oleoyl-glycero-3-phosphocholine; $\mathrm{BR}=$ Bacteriorhodopsin.
}

hydrophobic transmembrane protein composed of 248 amino acid residues [25,26]. Importantly, it acts as a light-driven proton pump, where, upon absorption of a green light photon $\left(\lambda_{\mathrm{BR}} \approx 568 \mathrm{~nm}\right)$, it undergoes conformational changes and pumps a proton out of the cell [27-29]. Absorption and release of a proton are two simultaneous events during the bacteriorhodopsin photocycle. In nature, the protein can be found organised in $2 \mathrm{D}$ crystalline patches of trimers assembled within the so called purple membrane of the bacterium Halobacterium salinarum. The protein has shown stability in the purple membrane at a wide range of $\mathrm{pH}$ values (2-10) and temperatures $\left(<96^{\circ} \mathrm{C}\right)[30,31]$. BR was chosen for the large wealth of existing literature on its structure in the purple membrane and because its activation should lead to increased fluctuations that are detectable with the surface scattering methods developed by our team.

An incorporation method based on the use of sugar-based detergent $\mathrm{n}$-dodecyl- $\beta$-D-maltopyranoside (DDM) was adapted from Refs [32-34] for inserting BR into floating bilayers. In order to characterize this insertion and demonstrate the activity of the protein, we have combined different experimental techniques. Quartz crystal microbalance with dissipation monitoring (QCMD) and fluorescence microscopy measurements enabled us to quantify the interactions between the protein and bilayers supported on surfaces, while AFM allowed us to obtain direct information on the insertion of proteins into the membrane. By using neutron (NR) and X-ray (XRR) reflectometry, we could access the structure of pristine bilayers and the structural and compositional modifications caused by protein incorporation and activation.

Double lipid bilayers are highly fragile systems, that need to be prepared by a combination of Langmuir-Blodgett and LangmuirSchaefer (LB-LS) techniques. Appropriate preparation requires the use of relatively large substrates and the bilayers to be kept hydrated at all times, rendering their manipulation during QCM$\mathrm{D}$ and AFM measurements near impossible. Consequently, we chose to optimize the incorporation protocol and confirm the insertion of the protein in single supported bilayers by using QCM-D and AFM techniques on supported bilayers prepared by vesicle fusion. Subsequently, we then demonstrated that the protocol could be applied to supported bilayers prepared by LB-LS via fluorescence microscopy, and studied the effect of BR insertion on scattering length density profiles by NR. This allowed us to validate the use of this technique for studying the insertion of bacteriorhodopsin. Finally, we investigated the insertion of BR in floating bilayers with fluorescence microscopy and neutron reflectivity, and demonstrated the activity of the protein with X-ray reflectivity experiments.

\section{Materials and methods}

\subsection{Materials}

Synthetic zwitterionic phospholipids as 1,2-distearoyl-snglycero-3-phosphocholine (DSPC) (purity >99\%), 1,2dipalmitoyl-sn-glycero-3-phosphocholine (DPPC) (purity >99\%) 
and 1-palmitoyl-2-oleoyl-glycero-3-phosphocholine (POPC) (purity $>99 \%$ ) as well as n-dodecyl- $\beta$-D-maltopyranoside (DDM) (purity $>99 \%$ and 1,2-dipalmitoyl-sn-glycero-3phosphoethanolamine-N-(7-nitro-2-1,3-benzoxadiazol-4-yl) (ammonium salt) (NBD-PE) (purity >99\%) were purchased from Avanti Polar Lipids (Lancaster, USA) and used without further purification. 5,5'-disulfato-1'-ethyl-3,3,3',3'-tetramethylindocarbo cyanine (Cy3) - Mono N-hydroxylsuccinimidyl (NHS) ester was purchased from Thermo Fisher Scientific Inc.. Pure water (resistivity $18.2 \mathrm{M} \Omega . \mathrm{cm}$ ) was obtained from a Millipore purification system. Heavy water $\left(D_{2} O\right.$, purity $\left.\geqslant 99.9 \%\right)$ and all solvents (chloroform (purity $\geqslant 99.9 \%$ ), ethanol (purity $\geqslant 99.8 \%$ ), acetone (purity $\geqslant 99.9 \%$ )) were purchased from Sigma-Aldrich (Saint-Quentin Fallavier, France) and used without further purification. BR was provided by the team of Valentin Gordeliy (Institut de Biologie Structurale, IBS, Grenoble, France); BR purification is described elsewhere [36].

Fluorescence microscopy experiments required labelling of lipid and protein molecules. Bilayers were labelled by adding $1 \%$ (by weight) of NBD-PE lipids to the solution in chloroform. BR molecules were labelled with Cy3 dye following a procedure adapted from [37] (see Supplementary Material). It is important to note that only the monolayer located further away from the solid support was labelled in supported lipid bilayers and double lipid bilayers. This allowed us to indirectly check the quality of the deposition in terms of a presence of a multilayered system. The fluorescent markers were chosen in such a way that two different microscopy filter cubes could be used to visualize separately either the lipid bilayer or protein molecules. Single and double lipid bilayers were prepared either by vesicle fusion or LB-LS deposition techniques. Details about sample preparation techniques and solid substrates used for all experiments are given in Sections S1 and S2 in the Supplementary Material.

\subsection{Protein incorporation method}

As mentioned above, in order to reconstitute the protein in large supported single and double lipid bilayers, a detergentmediated protein incorporation protocol, based on the destabilization of the bilayer by a sugar-based detergent, was adapted from Refs [32-34]. This protocol required an extensive optimization that was primarily carried out on supported lipid bilayers by means of QCM-D and AFM experiments. Details about these measurements are given in Section S5 of the Supplementary Material. Briefly, a solution of bacteriorhodopsin of predefined concentration in $0.05 \mathrm{mM}$ DDM was injected into the sample cell and left to incubate for 10-15 min, allowing detergent molecules to destabilize the bilayer and promote protein insertion. The concentration of protein solutions varied from 0.5 to $60 \mu \mathrm{g} / \mathrm{ml}$ depending on the experiment. The optimal concentration at which protein insertion was noticeable without compromising the quality of the lipid bilayer (in terms of surface coverage) was determined experimentally. For single bilayers the optimal concentration was selected to be from 1 to $10 \mu \mathrm{g} / \mathrm{ml}$, while for double lipid bilayers only concentrations of 0.5 and $1 \mu \mathrm{g} / \mathrm{ml}$ were used as, for larger concentration values, removal of lipids was observed. At the end of the incubation period, the sample was extensively rinsed with either water or buffer solutions to remove all unbound proteins and detergent molecules from the bulk phase. Flow rates of $1 \mathrm{ml} / \mathrm{min}, 0.15 \mathrm{ml} / \mathrm{min}$ and 0.5-1 $\mathrm{ml} / \mathrm{min}$ were used for NR and XRR, QCM-D and fluorescence microscopy experiments respectively.

\subsection{Atomic Force Microscopy}

Atomic Force Microscopy (AFM) measurements were performed on a Multimode 8, Nanoscope V (Bruker) operated in Peak Force
Tapping mode, with the force set point value of $\approx 300 \mathrm{pN}$ and a scan frequency of a 1-2 Hz [38]. A ScanAsyst-fluid probe was used for imaging (Bruker probes, Camarillo, CA, USA). Raw AFM images were leveled using the mean plan subtraction of order 1 with Gwyddion [39]. Due to the large stripe noise, AFM images were sent to the DeStripe server [40]. Finally, AFM images were flattened by using the Gwyddion median of differences method. Additional post-treatment was performed using a L-weight filter $[41,42]$. This Laplacian-based filter enhanced the visibility of single molecules and allowed us to distinguish individual proteins in a complex environment [43].

\subsection{Fluorescence Microscopy}

A Nikon TE-2000 inverted microscope equipped with a mercury light source (Nikon Instruments Inc., Japan) was used in epifluorescence mode for the fluorescence microscopy experiments. An oilimmersion objective LEICA with x100 magnification and a 1.3 numerical aperture was used. The temperature of the sample was controlled precisely with a heating chamber. The inverted microscope was equipped with two microscopy filter cubes, one for visualization and excitation of lipids (lipid channel, with filter wavelengths $482 \mathrm{~nm} / 506 \mathrm{~nm} / 536 \mathrm{~nm}$ ), and one for visualization and excitation of bacteriorhodopsin (BR channel, with wavelengths $543 \mathrm{~nm} / 562 \mathrm{~nm} / 593 \mathrm{~nm}$ ). Images were captured with a digital camera, Insight 18000, from Diagnostic Instrument. All images were recorded using software developed by André Schröder (Institut Charles Sadron, Strasbourg) with both the analysis and processing performed in Image [44].

\subsection{Reflectometry experiments}

\subsubsection{Specular reflectometry}

We have combined neutron and X-ray reflectometry to characterize our systems with a high spatial resolution down to a fraction of a nanometer. Specular reflectivity $R\left(q_{z}\right)$, defined as the ratio between the intensity of the reflected and incident beams (where $\theta$ is the angle of incidence and reflection and $\lambda$ is the wavelength of the beam), was measured as a function of $q_{z}$, the wave-vector transfer component perpendicular to the sample surface. For a schematic representation of the scattering geometry used see Fig. 1. This normal component of the wave-vector is defined as

$q_{z}=\frac{4 \pi}{\lambda} \sin \theta$

The result of the reflectivity data analysis is a scattering length or electron density profile (for NR and XRR respectively), which provides information about the internal composition and structure of the sample and can be described in terms of a finite number of flat layers (slab model) or in terms of a series of probability distribution functions [45]. In this work, the slab model was implemented (see Section 3 in Supplementary Material for further details) where, the $j$-th layer was characterized by a thickness, $d_{j}$, either scattering length density (SLD) or electron density (ED), $\rho_{j}$, and interface roughness, $\sigma_{j, j+1}$. The water volume fraction, $f_{j}^{s}$, in the layer can be determined from the total SLD value in NR experiments thanks to the contrast variation method $[46,47]$. Since scattering length and electron densities are proportional to the sum of neutron-nuclei scattering lengths and number of electrons in a given volume, respectively, both the nanoscale sample structure and the composition could be extracted upon modeling the data $[48,49]$. The structural parameters describing each layer in the slab model were optimised during the fitting procedure to obtain the best fit of the measured reflectivity data. The goodness of the fit was evaluated in terms of the minimum of the $\chi^{2}$ function (see Supplementary 


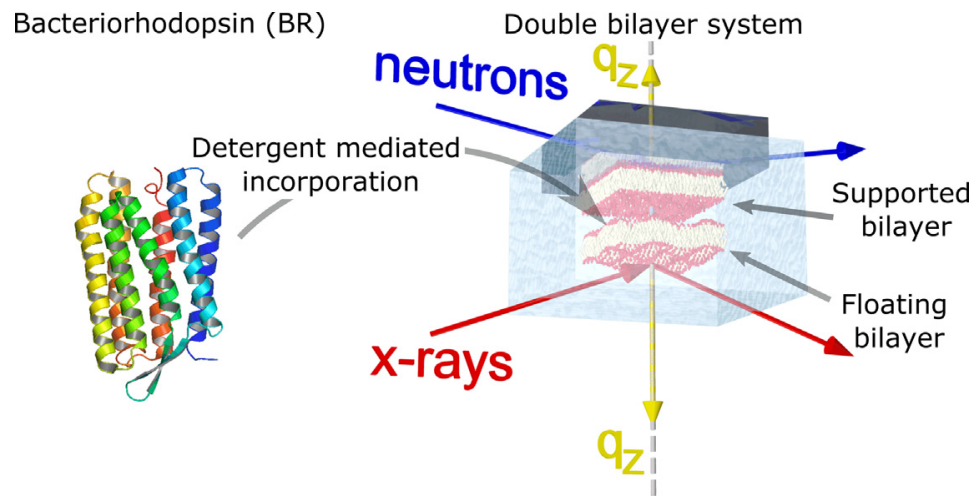

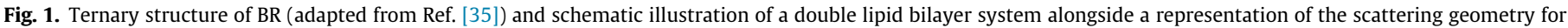
reflectometry experiments.

Material for the details). Data analysis was performed using the AuroreNR software [46].

\subsubsection{Neutron reflectometry}

NR experiments were performed on the reflectometers D17 [50] (Institut Laue-Langevin (ILL), Grenoble, France) and MARIA [51] (JCNS-MLZ, Garching, Germany). The D17 instrument was operated in time-of-flight mode, using wavelengths from 0.2 to $2.0 \mathrm{~nm}$. Based on Eq. (1), to cover a $q_{z}$-range from 0.085 to $3.5 \mathrm{~nm}^{-1}$, two angular configurations were used $\left(\theta=0.8^{\circ}\right.$ and $\left.\theta=3.2^{\circ}\right)$ with sample environment conditions following those presented in the literature [52]. MARIA, a monochromatic reflectometer, was utilized with two wavelengths, namely $0.5 \mathrm{~nm}$ and $1 \mathrm{~nm}$, selected by a rotating velocity selector, defining a $\Delta q_{z} / q_{z}=10 \%$ resolution. Incident and detector angles were varied in a $\theta-2 \theta$ configuration from $\theta=0.4^{\circ}$ to $4.5^{\circ}$ and the reflectivity curves were collected point by point. This allowed us to cover a $q_{z}$ range of $0.09 \mathrm{~nm}^{-1}$ to $2 \mathrm{~nm}^{-1}$. Since the sample angle was changed continuously, the slits' opening was adjusted at every angle to have a constant beam footprint at the sample position. Both instruments were operated with solid-liquid cells provided by the ILL. Sample temperature was controlled by a circulating water bath and kept constant at either $25^{\circ} \mathrm{C}$ or $48^{\circ} \mathrm{C}$ (depending on the experiment) during the measurements. Contrast variation [47] was used to improve the accuracy of the data modeling. The main contrasts used throughout the different experiments were $100 \% \mathrm{H}_{2} \mathrm{O}\left(\mathrm{SLD}=-0.56 \times 10^{-4} \mathrm{~nm}^{-2}\right)$, siliconmatch water (SiMW, i.e. $62 \% \mathrm{H}_{2} \mathrm{O}$ and $38 \% \mathrm{D}_{2} \mathrm{O}, \mathrm{SLD}=2.07 \times 10^{-4}$ $\mathrm{nm}^{-2}$ ), 4-match water (4 MW, i.e $34 \% \mathrm{H}_{2} \mathrm{O}$ and $66 \% \mathrm{D}_{2} \mathrm{O}$, $\left.\mathrm{SLD}=4 \times 10^{-4} \mathrm{~nm}^{-2}\right)$ and $100 \% \mathrm{D}_{2} \mathrm{O}\left(\mathrm{SLD}=6.35 \times 10^{-4} \mathrm{~nm}^{-2}\right)$, where $\mathrm{H}_{2} \mathrm{O}$ and $\mathrm{D}_{2} \mathrm{O}$ percentages refer to volume fractions. Data reduction was performed using the routines available at the large scale facilities.

\subsubsection{X-ray reflectometry}

$\mathrm{X}$-ray reflectometry measurements were performed at the SixS beamline of the SOLEIL synchrotron (Saint-Aubin, France). The Xray beam energy was fixed at $18.4 \mathrm{keV}(\lambda=0.0674 \mathrm{~nm})$ by means of a monochromator. Beam shape and size at the sample position were controlled using collimation slits. The divergence of the beam was $\approx 0.6 \cdot 10^{-3}$ degrees. The specular reflectivity $R\left(q_{z}\right)$ was measured by varying the incident angle $\theta$ from $0^{\circ}$ to $1.5^{\circ}$, which covered a $q_{z}$-range of $0 \mathrm{~nm}^{-1}$ to $5 \mathrm{~nm}^{-1}$. To avoid radiation damage, the sample was translated laterally by $3 \mathrm{~mm}$ after each reflectivity measurement to perform the next observation on a fresh area of the sample. The lateral width of the beam was fixed to 1 or $1.5 \mathrm{~mm}$ (depending on the sample) for every angle of incidence. The background scattering signal was measured by performing an "offset scan", i.e. by measuring the scattered intensity around the region of specular signal [53,54].

During X-ray reflectivity experiments, double lipid bilayer samples were measured before and after the injection of protein solution. After incubation, measurements were performed under two illumination conditions: i) in complete darkness, and ii) with green-yellow light (defined by a long-pass filter with $530 \pm 5 \mathrm{~nm}$ cut-on wavelengths [55]). This sequence of illumination conditions was applied twice on the same sample in order to test reversibility of any observed effects. The same measurements were performed also on a pristine double lipid bilayer prior to bacteriorhodopsin insertion in order to exclude the presence of any effect of the illumination on the bilayer structure. The light intensity directed onto the cell opening was estimated to be $\sim 0.05-0.1 \mathrm{~W} / \mathrm{cm}^{2}$. Data acquisition was performed using the routines available at the SixS beamline and data reduction implementing a custom made routine.

\subsubsection{Scattering length density and electron density profiles of $B R$}

The structure of bacteriorhodopsin trimer is very well known $[10,56]$, having been studied in particular with high-resolution Xray diffraction experiments $[57,58]$. From the position of the different atoms in a trimer, derived from the protein crystal structure reported in Protein Data Bank (PDB) code 1C3W [57], the onedimensional scattering length density and electron density profiles were calculated as follows: (i) the scattering length $b_{i}$ (or the electron number $n_{e, i}$ ) was assigned to each atom $i$; (ii) the 3D protein structure was sliced into slabs of thickness $\delta z=0.2 \mathrm{~nm}$, with cuts perpendicular to the axis of symmetry of the trimer (i.e. parallel to the plane of the bilayer); (iii) for each slab the scattering length density profiles $S L D_{\mathrm{BR}}(z)$ and the electron density $E D_{\mathrm{BR}}(z)$ were computed as:

$\operatorname{SLD}_{\mathrm{BR}}(z)=\frac{1}{\mathscr{A}_{\mathrm{BR}} \delta z} \sum_{i \in\{z, z+\delta z\}} b_{i}$,
$E D_{\mathrm{BR}}(z)=\frac{1}{\mathscr{A}_{\mathrm{BR}} \delta z} \sum_{i \in\{z, z+\delta z\}} n_{e, i}$,

where $\mathscr{A}_{\mathrm{BR}}$ is the area of the protein section $\left(\mathscr{A}_{\mathrm{BR}}=27 \pm 2 \mathrm{~nm}^{2}\right)$, estimated from the protein crystal structure shown in Fig. 3.

SLD and ED profiles of bacteriorhodopsin are presented in Section S3 in Supplementary Material. The profiles are slightly asymmetrical, due to the sensitivity the experiments, we were unable to distinguish a preferential orientation of the bacteriorhodospin in the supported membranes. Therefore, it was assumed that $50 \%$ of BR molecules were pointing up and 50\% were pointing down. 


\section{Results and discussion}

The main goal of this work was to reconstitute bacteriorhodopsin into the floating bilayer of a double lipid bilayer system, while preserving its structure and function of bacteriorhodopsin upon insertion. One of the main challenges was the use of large substrates for double lipid bilayer formation for NR and XRR experiments (40 and $25 \mathrm{~cm}^{2}$ ). In addition, the preparation of double lipid bilayers required the implementation of ad hoc bilayer deposition techniques for particular lipid composition. These requirements were sometimes incompatible with those of other characterization techniques employed. For instance, QCM-D cells could not be fully immersed in water because of the presence of water-sensitive components, making vesicle fusion the only usable technique to prepare lipid bilayers for this type of experiment. Since the instrument only allowed measurements within a maximum temperature of $\simeq 40^{\circ} \mathrm{C}$, the type of lipid molecules that could be used was limited to those which are in the fluid phase below that temperature. Similar constraints were present for other techniques and, for this reason, different lipid compositions had to be used. In this context, POPC supported lipid bilayers (fluid at room temperature) were used to optimize the reconstitution protocol for bacteriorhodopsin insertion into fluid single supported bilayers, while DPPC:POPC ones, showing coexistence of fluid (POPC) and gel (DPPC) domains at room temperature, were used to optimize and demonstrate protein reconstitution in both phases.

We first focused on simple supported lipid bilayers with the objective of optimizing the protocol of Milhiet and co-workers [32] that had been previously utilized for protein incorporation into supported bilayers of a maximum surface of $1 \mathrm{~cm}^{2}$. Here, supported lipid bilayers composed of POPC and DPPC:POPC (1:1 by mol) lipids of small surface area $\left(\simeq 1 \mathrm{~cm}^{2}\right)$ were investigated by QCM-D and AFM, allowing for the optimization of the experimental conditions for bacteriorhodopsin reconstitution on these small samples. The protocol was then transferred to DPPC and POPC supported lipid bilayers of larger areas for fluorescence microscopy and NR. Structural effects on the floating bilayer of double lipid bilayers caused by the activity of bacteriorhodopsin under illumination were finally investigated by XRR.

\subsection{Bacteriorhodopsin reconstitution in single lipid bilayers}

Formation of fluid supported lipid bilayers by vesicle fusion was routinely monitored by QCM-D experiments and all the bilayers prepared in this work presented frequency shifts and dissipation factors in agreement with those expected [59]. These supported lipid bilayers were exposed to solutions containing DDM with and without bacteriorhodopsin to optimize both detergent and protein concentrations as well as incubation time. QCM-D experiments showed that both bacteriorhodopsin interaction with supported lipid bilayers and reconstitution are fast processes where the duration of incubation period did not play a major role. To allow system equilibration an incubation time of 10-15 min for the detergent-protein mixtures in presence of a lipid bilayer was chosen here. QCM-D experiments demonstrated that the effect of 0.05 mM DDM solution on POPC supported lipid bilayers was negligible, as the lipid bilayer original rigidity and amount of lipids per unit area were preserved (see Section S5.2 in Supplementary Material). QCM-D measurements provided an indirect evidence of protein reconstitution into fluid-phase supported lipid bilayers as shown in Supplementary Material. No effect of the replacement of $\mathrm{H}_{2} \mathrm{O}$ by $\mathrm{D}_{2} \mathrm{O}$ in the aqueous phase on supported lipid bilayer formation and bacteriorhodopsin insertion was observed. Protein concentrations in the range $0.5-10 \mu \mathrm{g} / \mathrm{ml}$ in $0.05 \mathrm{mM}$ DDM buffer and water solution were found to be appropriate for the successful insertion into fluid supported lipid bilayers. Additional measurements by neutron reflectometry on supported lipid bilayers were performed to further optimize the quantity of protein inserted, by varying the concentration of the proteins in the injected solution. These experiments showed that an injection of a too concentrated bacteriorhodopsin solution could lead to a strong modification of the sample, with bilayer solubilisation, which was highly undesirable. Details about these measurements are given in the Supplementary Material Section S7.

Once the optimized conditions for protein reconstitution were found, AFM measurements were performed to investigate the insertion of bacteriorhodopsin in supported lipid bilayers in both fluid and gel-phase. In Fig. 2 an AFM topography image of DPPC: POPC supported lipid bilayer ( $1: 1$ by mol) upon bacteriorhodopsin insertion is shown. The sample imaging was performed at room temperature and domains formed by the coexistence of lipids in fluid-phase (POPC-rich) and in gel-phase (DPPC-rich) are clearly distinguishable. Height profiles corresponding to the marked regions are also shown. These profiles indicate that the thickness difference between DPPC-rich (thickness $\approx 5.1 \pm 0.2 \mathrm{~nm}$ ) and POPC-rich (thickness $\approx 3.8 \pm 0.2 \mathrm{~nm}$ ) domains is approximately $1 \mathrm{~nm}$. All these values are consistent with those reported in literature for bilayers composed of POPC and DPPC in the fluid and gel phase respectively [60,61].

Ring-shaped bright spots can be seen in both regions of the lipid bilayer as well as on the bare mica (Fig. 2(a)). On mica, a height $h_{\mathrm{AFM}}=4.5 \pm 0.2 \mathrm{~nm}$ is compatible with the height of the bacteriorhodopsin trimer determined from the analysis of its X-ray structure (PDB code 1C3W, [57]) (see Fig. 3) and in agreement with other studies of BR interactions with membranes [62,63]. These ring-shaped structures are more clearly visible in Fig. 2(d), where a patch of the gel phase DPPC-rich lipid bilayer with incorporated bacteriorhodopsin is shown on an enlarged scale. It should be noted that the incorporation of BR into DPPC highlights a patch which is higher than surrounding BR-free DPPC. Profiles drawn through the BR-rich DPPC patch (see Fig. 2(e-f)) show that an external part of the protein protrudes from $0.5 \mathrm{~nm}$ to $1.8 \mathrm{~nm}$ above the bilayer, demonstrating that the protein is inserted into the bilayer and not just deposited on it.

Our finding that BR protrudes out of the membrane by $\simeq 0.5 \pm$ $0.1 \mathrm{~nm}$ is in excellent agreement with previous studies ([64-66]). AFM experiments therefore clearly show the insertion of bacteriorhodopsin into the membrane along its symmetry axis. We notice also that the side of the molecule having the largest surface is often seen in contact with the substrate. AFM experiments have shown (Section S6.2 in Supplementary Material) that BR strongly adsorbs on the bare surface in the form of a trimer with the preferential orientation of pore-axis normal to the surface.

The lateral dimension of BR shows a diameter of about $22.0 \pm 2.5 \mathrm{~nm}$ (value averaged over 24 measurements) as observed on the L-weight filtered AFM image (see Fig. S6 in Section S6 in Supplementary Material). This lateral dimension is larger than the known crystal structure [57] (Fig. 3(a)), which can be due to a combination of an AFM tip-sample convolution effect and deformed BR trimer due to low compaction inside the membrane (low BR concentration) or on the mica.

NR experiments on an adsorbed protein layer on a bare surface were performed to determine the structure of bacteriorhodopsin in this condition and to complement AFM data. Reflectivity curves, measured in three contrast schemes $\left(\mathrm{D}_{2} \mathrm{O}\right.$, SiMW and $\left.\mathrm{H}_{2} \mathrm{O}\right)$, together with the best fits are shown in Fig. 3(c). The structural parameters obtained from the fits are summarized in Section S10 in Supplementary Material. The corresponding scattering length 

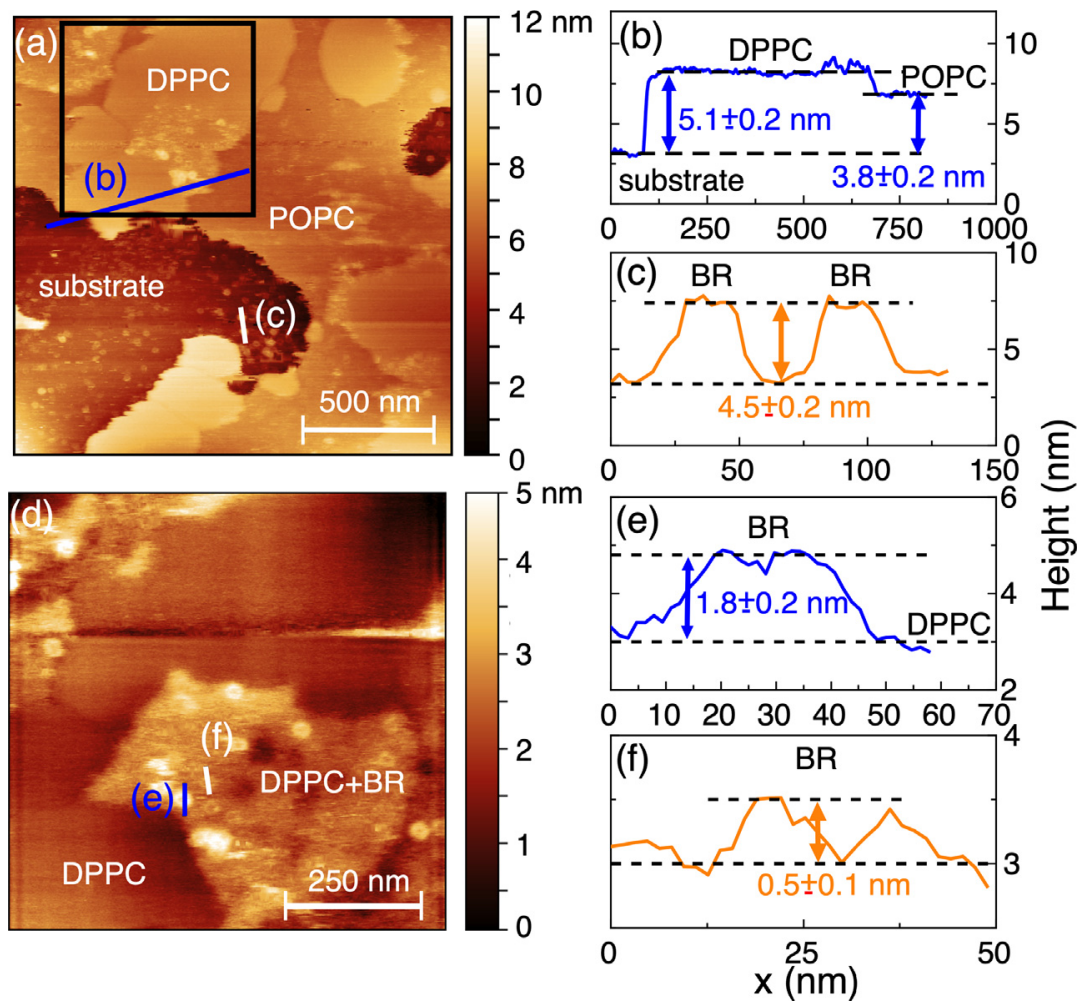

Fig. 2. (a) AFM images of a POPC:DPPC (1:1 by mol) supported lipid bilayer after BR reconstitution at room temperature. Image size is $1.66 \times 1.66 \mu \mathrm{m}^{2}, 512 \times 512 \mathrm{px}{ }^{2}$. (b) Height profile corresponding to the blue line in (a) indicating the substrate, DPPC-rich and POPC-rich domains. (c) Height profile corresponding to the white line in (a) consistent with BR trimers adsorbed on a mica surface. (d) Zoom on a DPPC-rich domain, black frame in image (a). Image size is $0.8 \times 0.8 \mu \mathrm{m}^{2}, 512 \times 512 \mathrm{px}^{2}$. (e) Height profile corresponding to the blue line in (d) indicating a partially inserted BR trimer in a DPPC-rich domain. (f) Height profile corresponding to the white line in (d) indicating a fully inserted BR trimer in a DPPC-rich domain.

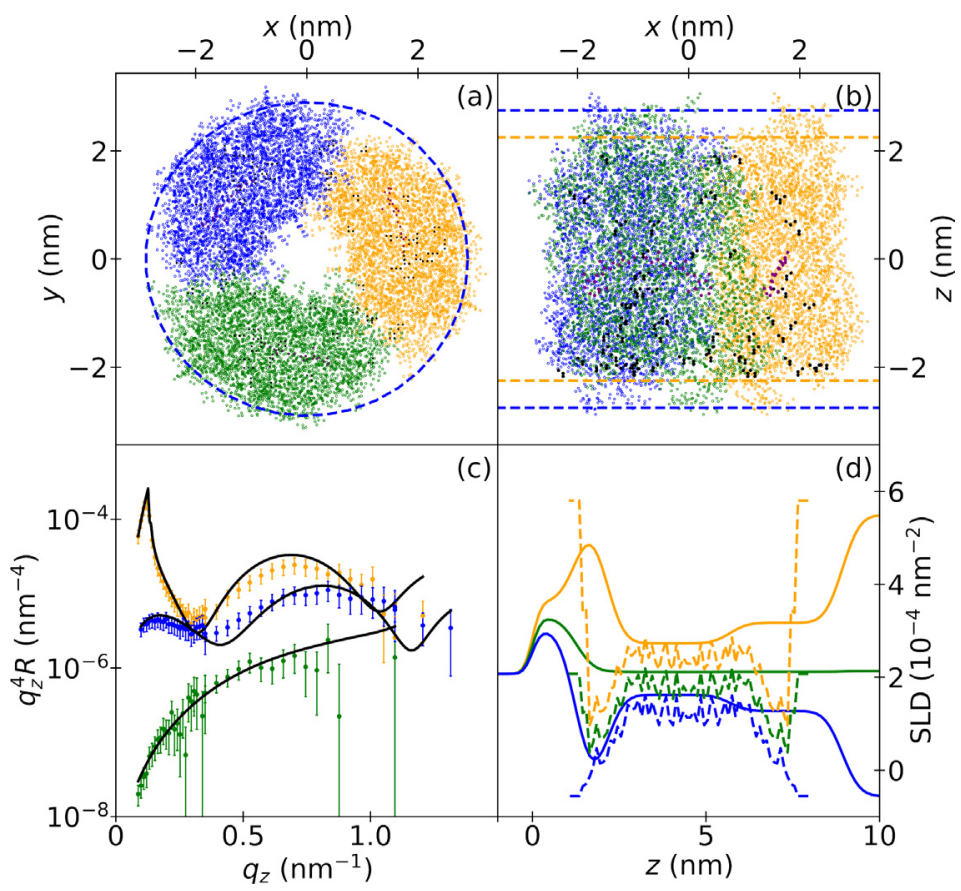

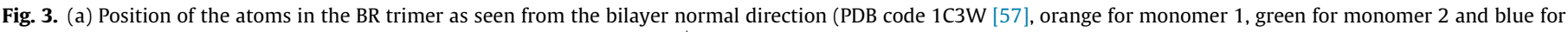



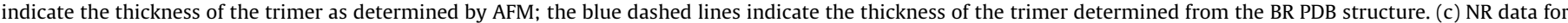

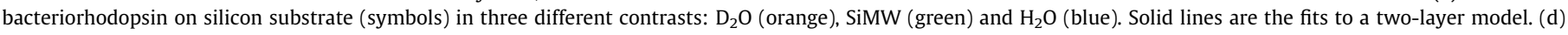

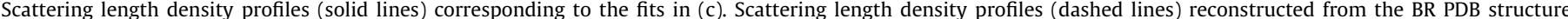

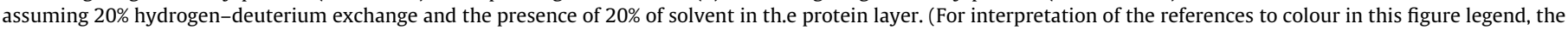
reader is referred to the web version of this article.) 
density profiles are shown as solid lines in Fig. 3(d). The latter indicates the presence of a first dense layer ( $82 \pm 1 \%$ of proteins) about $4 \mathrm{~nm}$ thick, and a second less dense layer on top of it, which is understood to correspond to incomplete protein adsorption. This result is in a good agreement with our AFM topography image of BR adsorbed on a bare substrate (see Section S6.2 in Supplementary Material). SLD profiles calculated from the PDB structure file [57] using Eq. (2) are shown as dashed lines in Fig. 3(d). The reconstituted SLD profiles describe a layer characterized by $80 \pm 10 \%$ of proteins (first layer) and are in good agreement with the profiles obtained from the modeling of NR data (solid lines in Fig. 3(d)). These results allowed us to confirm the formation of a dense protein layer on the silicon substrate, and to strongly constrain the scattering length density values that were used in the following analysis of the NR and XRR data from single and double bilayer systems.

Fluorescence microscopy experiments were performed on supported lipid bilayers deposited on glass slides by LB-LS techniques, to obtain complementary information on the lateral lipid bilayer structure at the microscale. In Fig. 4, fluorescence microscopy images for DPPC supported lipid bilayers before ( $a$ and $b$ ) and after (c and d) bacteriorhodopsin reconstitution are presented. The top images were obtained by using the lipid channel and the bottom images were obtained from the same sample by using the bacteriorhodopsin channel (for the definition of the channels see Materials and methods). In all cases, fluorescence profiles (orange for the lipids fluorescence signal, blue for the proteins) are shown. They correspond to the delimited areas in Fig. 4 and were obtained along a line passing through a defective region in the sample. Before insertion, the fluorescence level detected in the bacteriorhodopsin channel was very low ( $7 \pm 4$ a.u, close to the background noise), while that of the lipid channel reached $130 \pm 10$ a.u.. After protein insertion, the fluorescence level of the bacteriorhodopsin channel increased to $170 \pm 15$ a.u., while the lipid level remained constant at $132 \pm 15$ a.u. Interestingly, a strong signal originating from protein markers was detected in the defective region of the bilayer (spikes in the blue profile). This is further confirmation of the ability of bacteriorhodopsin to adsorb on the bare hydrophilic substrate. However, the majority of the bacteriorhodopsin signal was localized in bilayer-enriched regions of the sample, suggesting effective protein insertion in supported lipid bilayers in gel phase.

NR experiments on supported lipid bilayers composed of POPC lipids were also performed on the MARIA reflectometer to resolve the internal structure of fluid supported lipid bilayers at the molec- ular level both in the absence and in the presence of proteins. Protein reconstitution was performed by injecting a solution of $10 \mu \mathrm{g} /$ $\mathrm{ml} \mathrm{BR}$ in $0.05 \mathrm{mM}$ DDM $\mathrm{H}_{2} \mathrm{O}$ PBS buffer into the solid/liquid cell with the deposited POPC bilayer, incubating for $15 \mathrm{~min}$ and rinsing with $\mathrm{H}_{2} \mathrm{O}$ PBS buffer afterwards. Measured reflectivity curves, obtained best fits and scattering length density profiles are shown in Fig. 5(a) and (b). The structural parameters obtained from the fits are summarized in Section S10 in Supplementary Material. Changes in the SLD values of the tail regions $\left(-0.35 \times 10^{-4} \mathrm{~nm}^{-2}\right.$ for the pure POPC bilayer, $0.13 \times 10^{-4} \mathrm{~nm}^{-2}$ for the POPC bilayer with incorporated bacteriorhodopsin) indicate that, upon bacteriorhodopsin insertion, the final system is composed of $22 \pm 2 \%$ of bacteriorhodopsin. An increase of the water content in the tail regions upon protein reconstitution was also observed (from $10 \pm 2 \%$ to $35 \pm 5 \%$ ). The overall structure of the bilayer with bacteriorhodopsin is consistent with the known structure for pure POPC bilayers [67]. A more quantitative analysis was carried out by reconstructing the expected scattering length density profiles for a membrane embedding protein system by combining the SLD profile of pristine POPC bilayer with that of the bacteriorhodopsin, determined from the PDB atomic structure in accordance with

$S L D_{\text {bilayer+BR,calc }}=x_{p} S L D_{\mathrm{BR}}+x_{w} S L D_{w}+\left(1-x_{w}-x_{p}\right) S L D_{\text {bilayer }}$.

In Eq. (4), $S L D_{\mathrm{BR}}$ is the quantity calculated from the PDB structure as described in Materials and methods, $x_{p}$ and $x_{w}$ are the volume fractions of inserted protein and water molecules, respectively. The reconstructed scattering length density profiles are presented in Fig. 5(c) as solid lines assuming $x_{p}=20 \pm 5 \%$ and $x_{w}=30 \pm 10 \%$. These are in agreement with the scattering length density profiles determined from the modeling of NR data of the POPC bilayer after bacteriorhodopsin insertion. Finally, the possibility of formation of large domains of BR (as for example caused by direct adsorption on the substrate) was evaluated by modeling the NR data as a sum of two incoherent contributions originated from the POPC bilayer and from BR domains. The failure of such a modeling approach indicated that domain formation (and therefore preferential adsorption of BR on the substrate) larger than the coherence length $(<1 \mu \mathrm{m})$ can be disregarded, but we cannot rule out the formation of smaller domains.
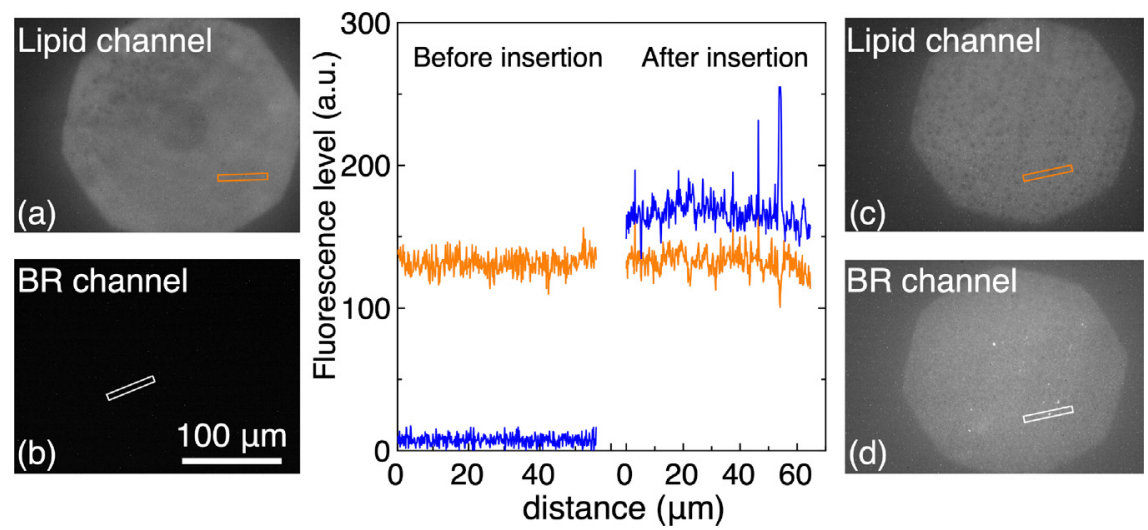

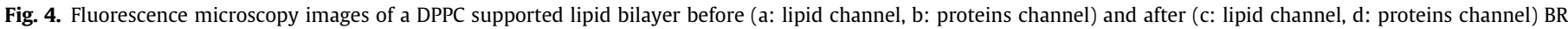

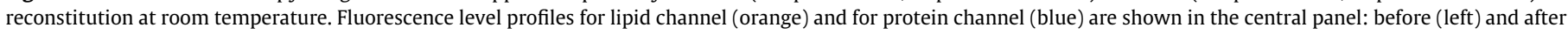

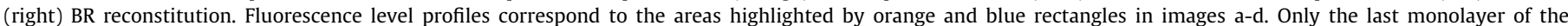

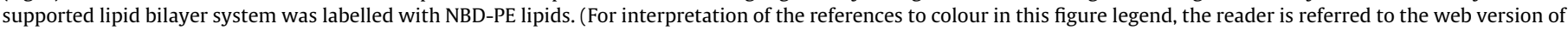
this article.) 


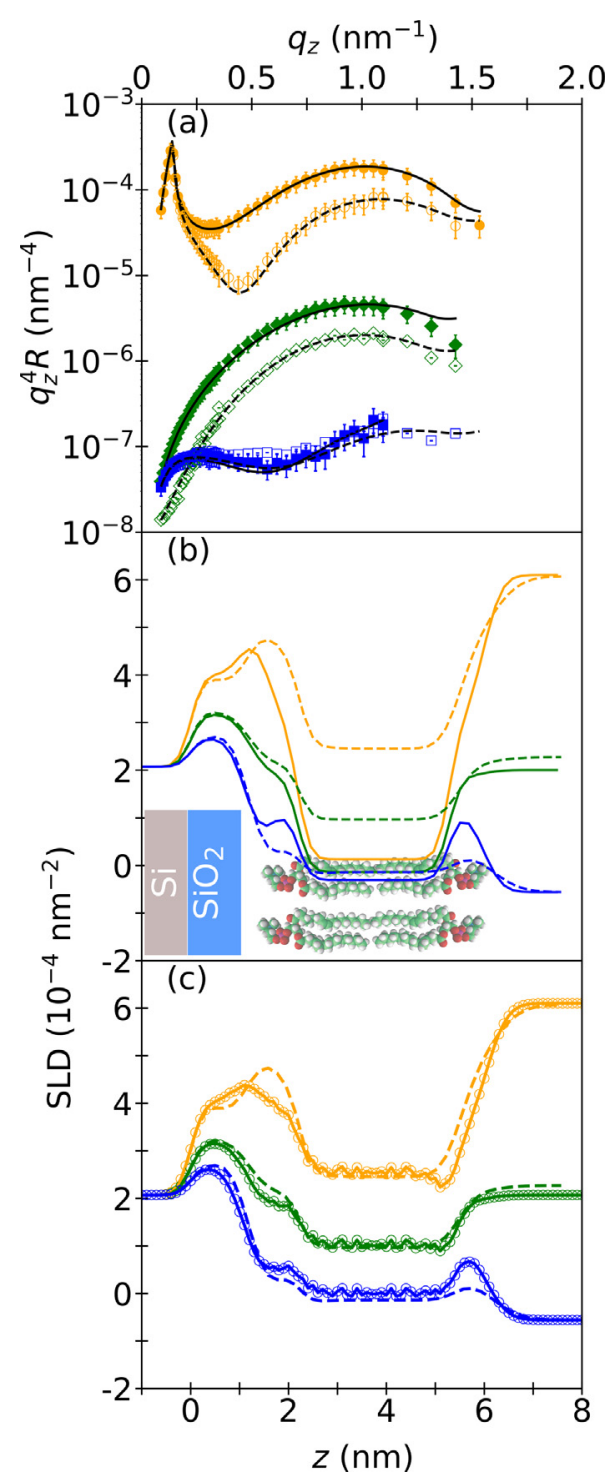

Fig. 5. (a) Neutron reflectivity curves of a POPC supported lipid bilayer at $T=25{ }^{\circ} \mathrm{C}$ before (closed symbols) and after (open symbols) BR reconstitution in three different contrasts: $\mathrm{D}_{2} \mathrm{O}$ (red), SiMW (green, shifted by $10^{-1}$ ) and $\mathrm{H}_{2} \mathrm{O}$ (blue, shifted by $10^{-2}$ ). (b) Scattering length density profiles corresponding to the fits before (solid lines) and after (dashed lines) BR reconstitution. (c) Scattering length density profiles reconstructed using Eq.4 (solid lines) compared to the experimental ones (dashed lines) obtained after BR insertion $\left(x_{p}=20 \pm 5 \%\right.$ and $x_{w}=30 \pm 10 \%$ ). (For interpretation of the references to colour in this figure legend, the reader is referred to the web version of this article.)

\subsection{Bacteriorhodopsin reconstitution in double lipid bilayers}

After confirming bacteriorhodopsin was inserted into supported lipid bilayers, floating lipid bilayers were used as a biologically relevant platform for protein reconstitution.

In Fig. 6 fluorescence microscopy images of a DSPC-DPPC double lipid bilayer before (a-b) and after (c-d) bacteriorhodopsin incorporation are shown. As illustrated for the supported lipid bilayers, the profiles (orange for the lipid channel, blue for the protein channel) were analyzed along a cut through a defective region in the floating bilayer. A control experiment was also performed on a double lipid bilayer prior to protein incorporation (Fig. 6a and b). In this case, only a very low signal was detected from the pristine sample on the bacteriorhodopsin channel (12 \pm 4 a.u.). After protein incorporation, the signal originating from the protein markers became visible ( $60 \pm 5$ a.u.), but it was nevertheless weaker than the one observed for supported lipid bilayers. This difference could be related to the different amount of protein inserted, but also to experimental conditions, such as exposure time and illuminated area, which might be slightly different for different experiments. In contrast to the supported lipid bilayer case, no bacteriorhodopsin accumulation in the defective regions of the floating bilayer was observed. This could indicate that proteins did not penetrate beneath the floating bilayer and no bacteriorhodopsin adsorption/incorporation occurred in the DSPC bilayer located beneath the floating one. The images also indicate that the interaction of bacteriorhodopsin with the double lipid bilayer did not drastically change its morphology at microscale. Moreover, since only the monolayer, which was farther away from the substrate, was fluorescently marked, fluorescence microscopy experiments showed that the overall structure of the double lipid bilayer was well preserved, at the microscale in the gel phase. However, these experiments could not exclude the presence of changes in the bilayer (such as the formation of small pores or defects) at the nanoscale due to limited instrument resolution. Despite using fluorescence microscopy, the difference between protein reconstitution, protein partial incorporation and protein adhesion to the lipid bilayer could not be distinguished due to the low spatial resolution of the technique. Moreover, the technique did not quantify the amount of bacteriorhodopsin inserted in the lipid bilayers. To overcome this limitation, specular NR and XRR experiments were performed. They allowed us to characterize the internal structure of the bilayer at the nanoscale, before and after protein reconstitution, as well as to quantify the amount and location of bacteriorhodopsin. NR experiments were performed on a DSPCDPPC double lipid bilayer at $48^{\circ} \mathrm{C}$, before and after protein reconstitution. The measurements at high temperature allowed to bring the floating DPPC bilayer into the fluid phase while keeping the supporting DSPC bilayer in gel phase. The experimental data, together with fits and scattering length density profiles are shown in Fig. 7. The protein reconstitution step was performed by injecting a solution of $0.5 \mu \mathrm{g} / \mathrm{ml}$ bacteriorhodopsin in $0.05 \mathrm{mM}$ DDM $\mathrm{H}_{2} \mathrm{O}$ PBS buffer into the cell, incubating for $15 \mathrm{~min}$ and rinsing with buffer afterwards. For a correct interpretation of the collected measurements it was crucial to investigate the effect of detergent molecules on the double lipid bilayer structure separately. As reported in Section S8 in Supplementary Material, only a minor and insignificant effect of $0.05 \mathrm{mM}$ DDM on a double lipid bilayer was observed, confirming that any variation in reflectivity, and thus, in scattering length density profiles, originated from bacteriorhodopsin.

The scattering length density profiles obtained from the analysis of the data confirmed a good quality of the pristine double lipid bilayer. After bacteriorhodopsin incorporation, no significant modifications of the first supported bilayer were detected, as presented in Fig. 7(b). The floating bilayer exhibits significant changes, consistent with those reported for the single bilayer case (see Fig. 5). As already described for the case of a single POPC bilayer, a quantitative analysis was performed by reconstituting the SLD profiles of the system after BR insertion using Eq. (4). SLD profiles for a double DSPC-DPPC bilayer after bacteriorhodopsin insertion were calculated by combining the SLD profiles of the pristine bilayer with the ones extracted from the PDB structure of a bacteriorhodopsin trimer. These reconstructed profiles are compared to the experimental ones in Fig. 7(c). Assuming a protein fraction of $x_{p}=25 \pm 10 \%$ and a water fraction of $x_{w}=20 \pm 5 \%$, Eq. (4) led to a very good agreement between calculated and experimental data, confirming the insertion of the bacteriorhodopsin in the floating bilayer and validating our modeling approach. A diffuse layer on top of the floating bilayer was also observed in the experimental 

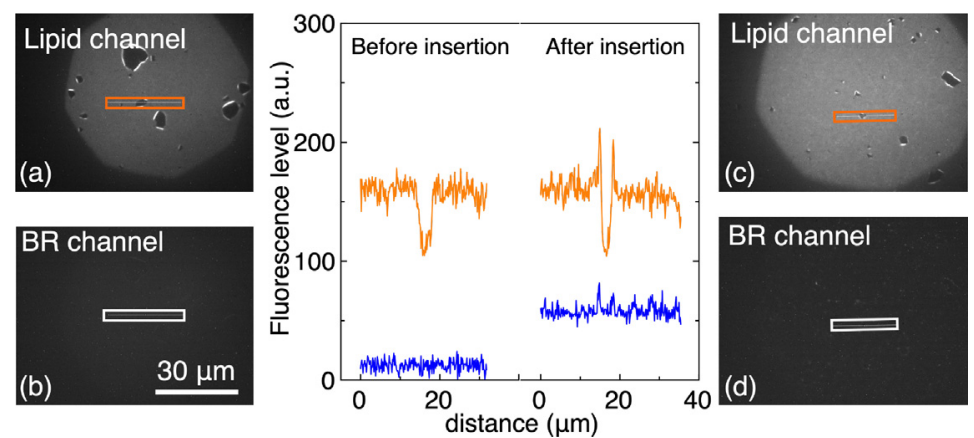

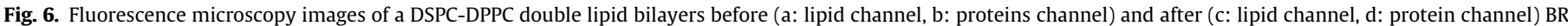

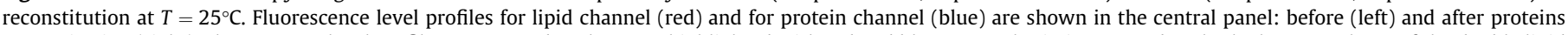

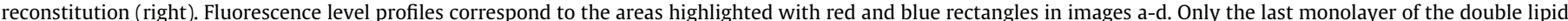

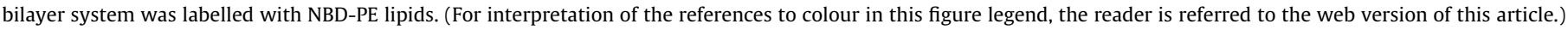

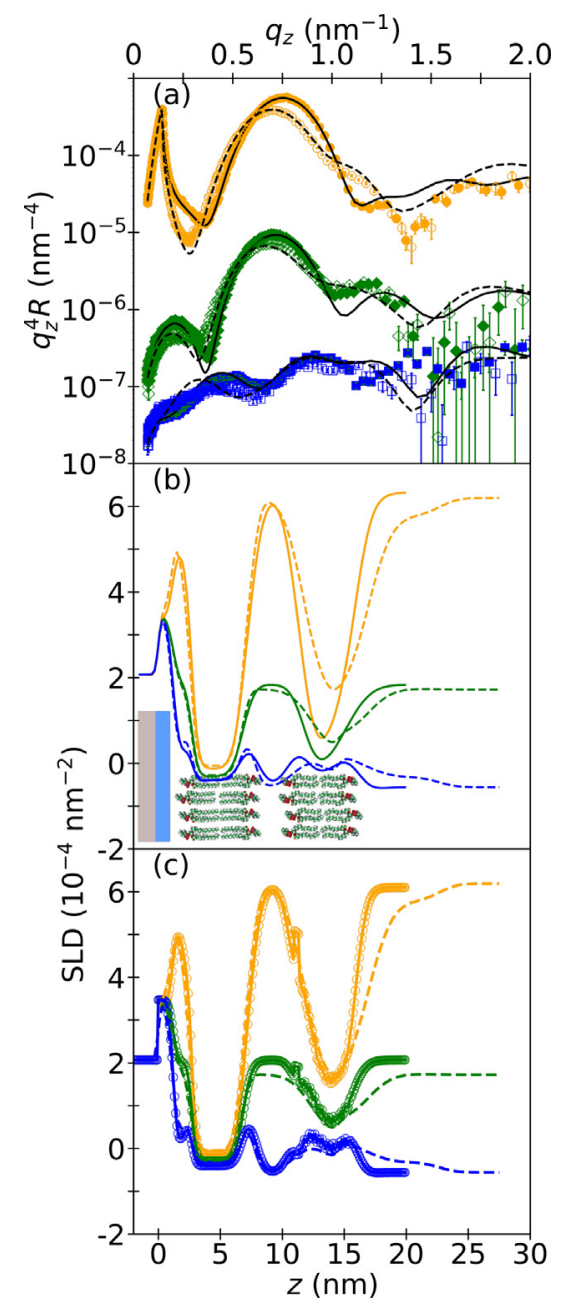

Fig. 7. (a) Neutron reflectivity curves for a DSPC-DPPC double lipid bilayer at $T=48^{\circ} \mathrm{C}$ before (closed symbols) and after (open symbols) BR reconstitution in three different contrasts: $\mathrm{D}_{2} \mathrm{O}$ (orange), SiMW (green, shifted by $10^{-1}$ ) and $\mathrm{H}_{2} \mathrm{O}$ (blue, shifted by $10^{-2}$ ). (b) Scattering length density profiles corresponding to the fits before (solid lines) and after (dashed lines) BR reconstitution. (c) Comparison between scattering length density profiles corresponding to the fits after BR reconstitution (dashed lines) and those reconstructed according to Eq. (4) (solid lines with empty circles). (For interpretation of the references to colour in this figure legend, the reader is referred to the web version of this article.) profiles. This was interpreted as a highly hydrated protein layer on top of the double lipid bilayer.

\subsection{Bacteriorhodopsin activity}

The optimal results, in terms of bilayer integrity and bacteriorhodopsin incorporation, were obtained for a double lipid bilayer in which both bilayers were in gel phase (DSPC-DSPC double lipid bilayer). This system was chosen for experiments in which the protein activity was triggered by illumination with visible light. X-ray reflectometry was the most suited technique [49] to investigate the structural changes induced in the double lipid bilayer system by bacteriorhodopsin activity, as it allows to probe bilayer modifications down to the fraction of a nanometer scale. The effect of bacteriorhodopsin activity was studied by alternating light-ON and light-OFF conditions while measuring XRR data.

XRR data for the pristine DSPC-DSPC double lipid bilayer, measured at $25^{\circ} \mathrm{C}$, are presented in Fig. 8(a) (blue symbols). The corresponding electron density profile is shown in Fig. 8(b) (blue line). The structural parameters obtained from the XRR data analysis and reported in Supplementary Material are in agreement with data already published on similar systems [15]. For protein reconstitution, $1 \mu \mathrm{g} / \mathrm{ml}$ of bacteriorhodopsin in $0.05 \mathrm{mM}$ DDM $\mathrm{H}_{2} \mathrm{O}$ PBS buffer solution was injected into the sample cell, incubated for $10 \mathrm{~min}$ and then rinsed with PBS buffer. The reflectivity curve measured after protein reconstitution is shown in Fig. 8(a) (orange symbols). The structural and compositional changes in the supporting bilayer are not significant as seen from the electron density profiles and probably unrelated to the protein insertion, but rather linked to changes of water content in the chain region of the bilayer. To confirm the results obtained using a slab model, the more quantitative analysis based on Eq. (4) was performed following the approach already described for NR data. In Eq. (4), SLD values were replaced by electron density values. The reconstructed profiles are shown in Fig. 8(c) (black line). The modifications of the electron density profile were treated using the same approach as for analysis of SLD profiles obtained with NR. The two experimental techniques demonstrated a very consistent agreement as similar protein fractions $\left(x_{p}=20 \pm 5 \%\right)$ and water content $\left(x_{w}=40 \pm 10 \%\right)$ in the floating bilayer were obtained for both samples, despite their variabilities. These results are in good agreement with the neutron reflectivity experiments. A decrease in the overall thickness of the floating bilayer (from $5.7 \pm 0.3$ to $5.2 \pm 0.3$ $\mathrm{nm}$ ) and an increase in the thickness of the water layer between the two bilayers $\left(d_{w, 2}=1.2 \pm 0.2\right.$ vs. $d_{w, 2}=1.7 \pm 0.2 \mathrm{~nm}$ ) were also observed upon bacteriorhodopsin insertion, while the roughness parameters were almost unaffected. 


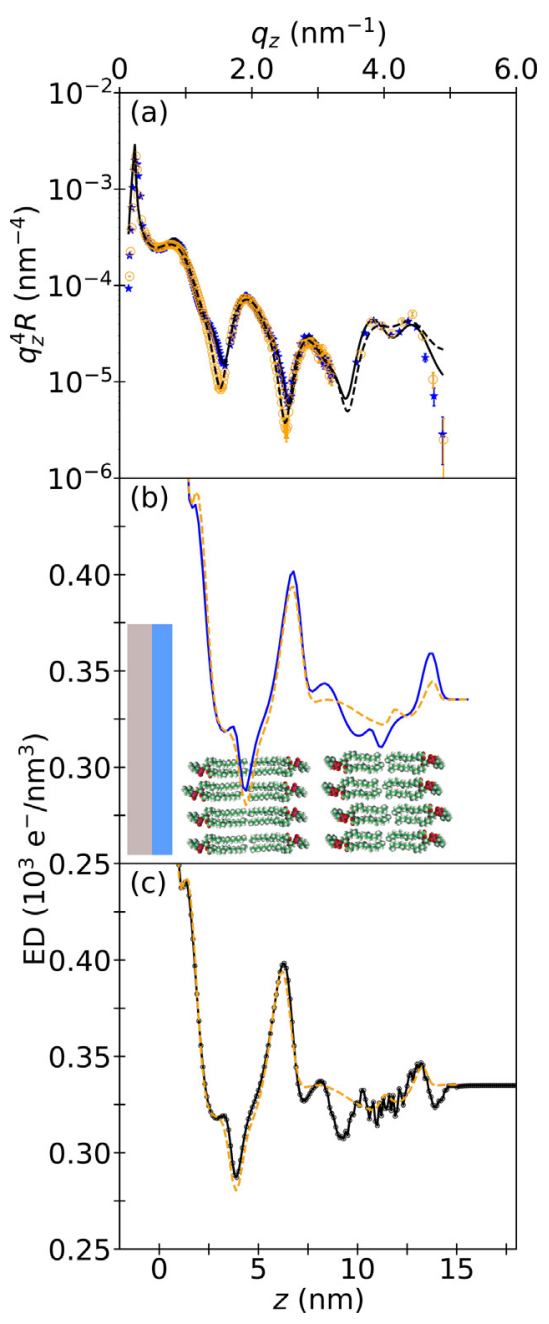

Fig. 8. (a) Specular X-ray reflectivity data and fits for a DSPC-DSPC double lipid bilayer measured at $25{ }^{\circ} \mathrm{C}$ before (filled blue symbols) and after (open orange symbols) BR incorporation. (b) ED profiles corresponding to the best fits shown in (a) (same color code). (c) ED profile for the sample after BR insertion (orange dashed lines) and the reconstructed ED profile according to Eq. (4) (black line). (For interpretation of the references to colour in this figure legend, the reader is referred to the web version of this article.)

The sample was illuminated with visible light to trigger $\mathrm{BR}$ activity. A high-pass filter with a $530 \pm 5 \mathrm{~nm}$ cut-on wavelength was used to select the appropriate spectrum for activation [68]. Light-ON XRR measurements were performed following the same procedure used for light-OFF measurements. It is worth mentioning that, as reported in [55], light was switched on 15 min before the XRR measurements to allow BR activity to reach equilibrium. The reflectivity curve corresponding to this condition is shown in Fig. 9(a) (orange filled symbols). By comparing the collected reflectivity curves before and after illumination, changes in the scattering signal, and therefore in the lipid bilayer structure, were detected after BR activation. Cyclical XRR measurements in light-ON and light-OFF conditions were performed to check the reversibility of this effect. Exposure to light was stopped and the sample was measured again after a resting period of at least $1 \mathrm{~h}$ to allow bacteriorhodopsin to go back to the dark-adapted state (blue open circles in Fig. 9(b)). This reflectivity curve (2nd dark state) overlaps exactly with the one measured during the first dark state, indicating a full structural recovery in the absence of protein activity. Light-ON measurements were also repeated and also in this case full reversibility in the specular XRR signal was observed (see Fig. 9(b)). A blank experiment on a pristine DSPC-DSPC double

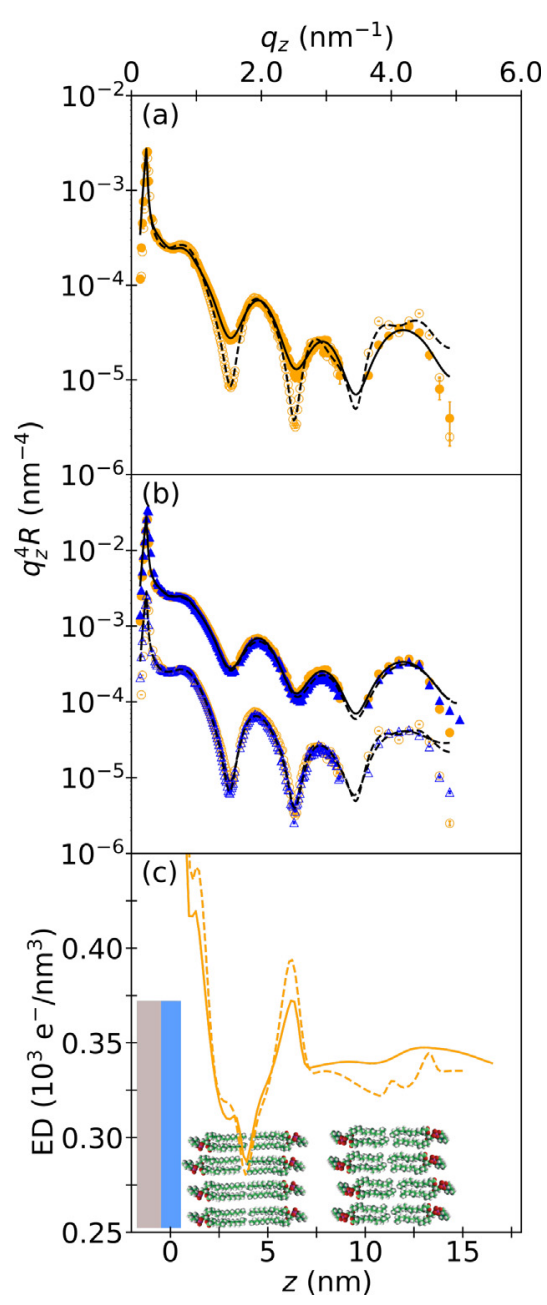

Fig. 9. Specular X-ray reflectivity data and fits for a DSPC-DSPC double lipid bilayer at $\mathrm{T}=25^{\circ} \mathrm{C}$ after BR incorporation: (a) light-OFF (open symbols) and light-ON (filled symbols) conditions; (b) test of reversibility: orange symbols correspond to the first illumination, blue symbols correspond to the second illumination experiment. Reflectivity curves for the light-ON measurements are shifted by a factor 10 for clarity. (c) ED profiles corresponding to the fits of the reflectivity curves collected during the first illumination experiment. ED profiles corresponding to the fits of reflectivity curves collected during the second illumination experiment are overlapping within errors with those corresponding to the first experiments, therefore they are not shown for clarity. (For interpretation of the references to colour in this figure legend, the reader is referred to the web version of this article.)

lipid bilayer was performed in order to test the effect of light on its structure (see Section S9 in Supplementary Material). Reflectivity measurements were performed in light-OFF and light-ON conditions, using the same visible wavelengths used for BR activation. No changes in reflectivity curves were observed, indicating no structural modifications of the floating bilayer. Therefore, all modifications upon illumination of the BR embedding sample were induced by the protein conformational changes and its pumping activity.

Bacteriorhodopsin activity caused significant reversible modifications of the floating bilayer (see electron density profile in Fig. 9 (b)). The main effect was a large increase in the lipid bilayer roughness from $\sigma_{\mathrm{OFF}}=0.2 \pm 0.2 \mathrm{~nm}$ to $\sigma_{\mathrm{ON}}=1.5 \pm 0.2 \mathrm{~nm}$. This increase is too large to be fully attributed to conformational changes of the $\mathrm{BR}$ as outward movements of the helixes $\mathrm{F}$ and an inwards movement of helix C towards the Schiff base during the BR photocycle [69]. There is most likely a contribution associated to an increase in bilayer fluctuations as predicted by theoretical work [70]. Mainly because of this increase in roughness, it is difficult to mea- 
sure with accuracy the structural changes in the floating bilayer. There is a significant increase in the total "water layer plus floating bilayer" thickness, of the order of $1.5 \mathrm{~nm}\left(d_{\mathrm{B}, \text { floating }}+d_{\mathrm{w}}=6.9 \pm 0.4\right.$ $\mathrm{nm}$ to $8.4 \pm 0.4 \mathrm{~nm}$ ), although it is difficult to discriminate precisely the contribution from each of the two layers. It is unlikely that this increase can be entirely attributed to a change in the conformation of the protein (contribution less than $0.5 \mathrm{~nm}$ according to Shibata et al. [71]) and there is most certainly an increase in water thickness which seems to correlate with the increase in roughness. The increase in the related roughness parameters by approximately $1 \mathrm{~nm}$ (see Table S10) is a very strong indication of the insertion of proteins into the membrane. This is not observed for double bilayers without bacteriorhodopsin (table S11) and well beyond what could be expected for bacteriorhodopsin patches directly adsorbed onto the bilayer or the substrate. Indeed, according to protein data bank data $(5 \mathrm{H} 2 \mathrm{H}$ to $5 \mathrm{H} 2 \mathrm{P}$ [29]) taken at different delay times ranging from 40 ns to $657 \mu \mu$ s after illumination, unit cell parameters ( $\mathrm{P}_{3}$ symmetry group) increase by less than $0.2 \mathrm{~nm}$. Taking into account relative coverage of double bilayer and bacteriorhodopsin patches, this would lead in a negligible increase in roughness (well below the o\&bseved $1 \mathrm{~nm}$ ) unless bacteriorhodopsin conformational changes trigger bilayer movement.

The work of Giahi et al. [72] on supported lipid multilayers with inserted BR had not revealed any effect of the protein's activity. Under similar conditions, we were able to observe significant modifications associated with BR activity on the structure and fluctuations of floating bilayers, probably because they are able to float freely near the substrate without being confined. The observed effects are consistent with small changes in the membrane structure and with an increase in membrane fluctuations, but it is impossible to separate these different contributions. Nonspecular reflectivity experiments, by providing access to quantitative information on the spectrum of membrane fluctuations, could help to clarify these different contributions and to test theoretical models.

\section{Conclusions and perspectives}

A robust and reproducible protocol for bacteriorhodopsin reconstitution into planar solid-supported single and floating phospholipid bilayers in gel and fluid phases was developed. A wide range of surface-sensitive techniques such as QCM-D, AFM, fluorescence microscopy, NR and XRR was used in order to confirm and optimize bacteriorhodopsin incorporation. Incubation time, protein and detergent concentration as well as lipid composition were optimized in order to preserve membrane structural integrity and protein activity. It was found that the optimal conditions involved the injection of $1 \mu \mathrm{g} / \mathrm{ml}$ of bacteriorhodopsin in $0.05 \mathrm{mM}$ DDM PBS buffer solution, incubation for $10 \mathrm{~min}$ followed by thoroughly rinsing with PBS buffer, on a previously formed lipid bilayer on a solid substrate. The structure and composition of the studied systems before and after bacteriorhodopsin insertion and activation were resolved at the nanoscale by means of specular NR and XRR experiments. The reversible effect of bacteriorhodopsin activity on the lipid bilayer structure was clearly demonstrated.

In particular, changes in the electron and scattering length density values upon bacteriorhodopsin reconstitution and the increase of the roughness of the floating bilayer upon sample illumination were consistent with an enhancement of out-of-equilibrium membrane fluctuations induced by the light-activated protein pumping activity. A complete understanding of the mechanisms at play during active membrane fluctuations requires a fine characterization of the fluctuation spectrum at the nanoscale. Floating lipid bilayers are excellent model systems to probe this phenomenon.
This is the first time that BR is reconstituted in floating bilayers. Work can be found in literature on reconstitution of different proteins in tethered bilayers $[73,74]$ but the interest here lies in the possibility to perform fluctuation studies on floating layers which are more difficult in the presence of tethers that may influence the fluctuations by pinning the membrane.

While NR and specular XRR experiments provided information about the structural and compositional changes of a bilayer caused by protein incorporation and activation with sub-nanometer resolution, off-specular XRR experiments will allow to measure the active membrane fluctuation spectrum induced by the protein pumping activity at the nanoscale. Combined analysis of specular and off-specular XRR experiments will provide the possibility to directly access the physical properties of the system such as bending modulus, surface tension and interaction potential between adjacent membranes [53,54]. Indeed, this work opens new outstanding perspectives as the investigation of the active fluctuation spectrum of DSPC-DSPC double lipid bilayer system with embedded bacteriorhodopsin.

\section{CRediT author statement}

Tetiana Mukhina: Methodology, Validation, Formal analysis, Visualization, Investigation, Writing - Original Draft, Writing Review and Editing. Yuri Gerelli: Methodology, Formal analysis, Software, Supervision, Investigation, Writing - Original Draft, Writing - Review and Editing, Resources. Arnaud Hemmerle: Investigation, Writing - Review and Editing. Alexandros Koutsioubas: Investigation. Kirill Kovalev: Resources. Jean-Marie Teulon: Formal analysis, Investigation. Jean-Luc Pellequer: Formal analysis, Investigation, Writing - Review and Editing. Jean Daillant: Conceptualization, Investigation, Software, Writing - Review and Editing. Thierry Charitat: Conceptualization, Methodology, Formal analysis, Writing - Original Draft, Writing - Review and Editing, Visualization, Supervision, Project administration, Funding acquisition. Giovanna Fragneto: Conceptualization, Methodology, Supervision, Writing - Review and Editing, Writing - Original Draft, Resources, Project administration, Funding acquisition.

\section{Declaration of Competing Interest}

The authors declare that they have no known competing financial interests or personal relationships that could have appeared to influence the work reported in this paper.

\section{Acknowledgements}

We acknowledge the Institut Laue-Langevin (ILL, Grenoble, France) (doi: 10.5291/ILL-DATA.8-02-803, doi: 10.5291/ILL-DATA. 9-13-689), SOLEIL synchrotron (Saint-Aubin, France), JCNS-MLZ (Garching, Germany) for the beamtime allocations and technical support. We acknowledge the D17 team (ILL), Alessandro Coati and Benjamin Voisin (SixS, SOLEIL synchrotron, Saint-Aubin, France) and MARIA team (JCNS-MLZ, Garching, Germany) for assistance during the experiments. We are very grateful to Kalvin Buckley from ILL for the development of sample environment cells and heating chamber for the X-ray reflectometry experiments. We acknowledge the group of Valentin Gordeliy (IBS, Grenoble, France) for providing the bacteriorhodopsin protein solutions. We are grateful to Daniel Lévy and Patricia Bassereau for very interesting and fruitful discussions. Jean-Paul Rieu (Institut Lumière Matière, Lyon, France) is acknowledged for help and assistance in AFM measurements. This work acknowledges the AFM platform at the IBS and we thank Shu-wen W. Chen for her help in the AFM image processing using the L-weight filter. We acknowledge André Schröder 
and Patrick Allgayer (ICS, Strasbourg, France) for the provided support during fluorescence microscopy experiments and help in designing and manufacturing the fluorescence microscopy sample cell. We acknowledge Anne Martel for her assistance with the fluorescent labelling of proteins, the Partnership for Soft Condensed Matter (PSCM) laboratories for providing facilities for sample preparation and characterization sample environment development and Samuel Winnall for English editing of the manuscript, all from the ILL. IBS acknowledges integration into the Interdisciplinary Research Institute of Grenoble (IRIG, CEA).

\section{Appendix A. Supplementary material}

Supplementary data associated with this article can be found, in the online version, at https://doi.org/10.1016/j.jcis.2021.03.155.

\section{References}

[1] R. Parthasarathy, J.T. Groves, Protein patterns at lipid bilayer junctions, Proc. Nat. Acad. Sci. 101 (35) (2004) 12798-12803, https://doi.org/10.1073/ pnas.0402168101.

[2] A. Luchini, Y. Gerelli, G. Fragneto, T. Nylander, G.K. Pálsson, M.S. Appavou, L. Paduano, Neutron Reflectometry reveals the interaction between functionalized SPIONs and the surface of lipid bilayers, Colloids Surf., B 151 (2017) 76-87, https://doi.org/10.1016/j.colsurfb.2016.12.005.

[3] M. Schulz, A. Olubummo, W.H. Binder, Beyond the lipid-bilayer: interaction of polymers and nanoparticles with membranes, Soft Matter 8 (2012) 48494864, https://doi.org/10.1039/C2SM06999G.

[4] E.T. Castellana, P.S. Cremer, Solid supported lipid bilayers: From biophysical studies to sensor design, Surf. Sci. Rep. 61 (10) (2006) 429-444, https://doi.org/ 10.1016/j.surfrep.2006.06.001.

[5] E. Sackmann, Supported membranes: Scientific and practical applications, $\begin{array}{lllll}\text { Science } & 271 & \text { (5245) } & \text { (1996) 43-48, https://doi.org/ }\end{array}$ 10.1126/science.271.5245.43.

[6] O.G. Mouritsen, O.S. Andersen (Eds.), In search of a new biomembrane model, no. 49 in Biologiske skrifter, Biol. Skr. Dan. Vid. Selsk., Copenhagen, 1998.

[7] V. Kiessling, A.J.B. Kreutzberger, B. Liang, P. Seelheim, J.D. Castle, D.S. Cafiso, L. K. Tamm, A molecular mechanism for calcium-mediated synaptotagmintriggered exocytosis, Nature Struct. Mol. Biol. 25 (10) (2018) 911-917, https:// doi.org/10.1038/s41594-018-0130-9.

[8] A. Beber, C. Taveneau, M. Nania, F.-C. Tsai, A. Di Cicco, P. Bassereau, D. Lévy, J.T. Cabral, H. Isambert, S. Mangenot, A. Bertin, Membrane reshaping by micrometric curvature sensitive septin filaments, Nature Commun. 10 (1) (2019) 420, https://doi.org/10.1038/s41467-019-08344-5.

[9] H.G. Franquelim, H. Dietz, P. Schwille, Reversible membrane deformations by straight DNA origami filaments, Soft Matter 17 (2021) 276-287, https://doi. org/10.1039/D0SM00150C.

[10] D.J. Muller, A. Engel, Strategies to prepare and characterize native membrane proteins and protein membranes by AFM, Curr. Opin. Colloid Interface Sci. 13 (5) (2008) 338-350, https://doi.org/10.1016/j.cocis.2007.09.002.

[11] A.Y. Pollitt, N.S. Poulter, E. Gitz, L. Navarro-Nuñez, Y.-J. Wang, C.E. Hughes, S.G. Thomas, B. Nieswandt, M.R. Douglas, D.M. Owen, D.G. Jackson, M.L. Dustin, S.P. Watson, Syk and Src family kinases regulate C-type lectin receptor 2 (clec-2)mediated clustering of podoplanin and platelet adhesion to lymphatic endothelial cells, J. Biol. Chem. 289 (52) (2014) 35695-35710, https://doi. org/10.1074/jbc.M114.584284.

[12] J. Katsaras, T. Gutberlet, Lipid Bilayers: Structure and Interactions, Springer Science \& Business Media, 2013.

[13] M.L. Wagner, L.K. Tamm, Tethered polymer-supported planar lipid bilayers for reconstitution of integral membrane proteins: Silane-polyethyleneglycol-lipid as a cushion and covalent linker, Biophys. J . 79 (3) (2000) 1400-1414, https:// doi.org/10.1016/S0006-3495(00)76392-2.

[14] E.-K. Sinner, W. Knoll, Functional tethered membranes, Curr. Opin. Chem. Biol. 5 (6) (2001) 705-711, https://doi.org/10.1016/S1367-5931(01)00269-1.

[15] T. Charitat, E. Bellet-Amalric, G. Fragneto, F. Graner, Adsorbed and free lipid bilayers at the solid-liquid interface, Eur. Phys. J. B - Condens. Matter Complex Syst. 8 (4) (1999) 583-593, https://doi.org/10.1007/s100510050725.

[16] G. Fragneto-Cusani, Neutron reflectivity at the solid/liquid interface: examples of applications in biophysics, J. Phys.: Condens. Matter 13 (21) (2001) 49734989, https://doi.org/10.1088/0953-8984/13/21/322.

[17] G. Fragneto, T. Charitat, F. Graner, K. Mecke, L. Perino-Gallice, E. Bellet-Amalric, A fluid floating bilayer, Europhys. Lett. 53 (1) (2001) 100-106, https://doi.org/ 10.1209/epl/i2001-00129-8.

[18] A. Hemmerle, G. Fragneto, J. Daillant, T. Charitat, Reduction in tension and stiffening of lipid membranes in an electric field revealed by X-ray scattering, Phys. Rev. Lett. 116 (22) (2016) 228101, https://doi.org/10.1103/ PhysRevLett.116.228101.

[19] F. Lolicato, L. Joly, H. Martinez-Seara, G. Fragneto, E. Scoppola, F. Baldelli Bombelli, I. Vattulainen, J. Akola, M. Maccarini, The role of temperature and lipid charge on intake/uptake of cationic gold nanoparticles into lipid bilayers, Small 15 (23) (2019) 1805046, https://doi.org/10.1002/smll.201805046.

[20] T. Mukhina, Active fluctuations and electrostatic interactions in floating lipid membranes Theses, Université de Strasbourg, 2019. https://tel.archivesouvertes.fr/tel-02468867.

[21] W.C. Wong, J.-Y. Juo, C.-H. Lin, Y.-H. Liao, C.-Y. Cheng, C.-L. Hsieh, Characterization of single-protein dynamics in polymer-cushioned lipid bilayers derived from cell plasma membranes, J. Phys. Chem. B 123 (30) (2019) 6492-6504, https://doi.org/10.1021/acs.jpcb.9b03789.

[22] G. Fragneto, T. Charitat, J. Daillant, Floating lipid bilayers: models for physics and biology, Eur. Biophys. J. 41 (10) (2012) 863-874, https://doi.org/10.1007/ s00249-012-0834-4.

[23] J. Prost, R. Bruinsma, Shape fluctuations of active membranes, Europhys. Lett. 33 (4) (1996) 321-326, https://doi.org/10.1209/epl/i1996-00340-1.

[24] D. Lacoste, P. Bassereau, An update on active membranes, in: G. Pabst, N. Kucerka, M.-P. Nieh, J. Katsaras (Eds.), Liposomes Liposomes, Lipid Bilayers and Model Membranes, CRC Press, Boston, MA, 2014, pp. 271-288, https://doi. $\operatorname{org} / 10.1201 / \mathrm{b} 16617$.

[25] M. Luckey, Membrane Structural Biology: With Biochemical and Biophysical Foundations, Cambridge University Press, 2014. ISBN 9781107030633.

[26] E. London, H.G. Khorana, Denaturation and renaturation of bacteriorhodopsin in detergents and lipid-detergent mixtures, J. Biol. Chem. 257 (12) (1982) 7003-7011, https://doi.org/10.1016/S0021-9258(18)34529-0.

[27] R. Neutze, E. Pebay-Peyroula, K. Edman, A. Royant, J. Navarro, E.M. Landau, Bacteriorhodopsin: a high-resolution structural view of vectorial proton transport, Biochimica et Biophysica Acta (BBA) - Biomembranes 1565 (2). https://doi.org/10.1016/s0005-2736(02)00566-7.

[28] H. Luecke, Atomic resolution structures of bacteriorhodopsin photocycle intermediates: the role of discrete water molecules in the function of this light-driven ion pump, Biochimica et Biophysica Acta (BBA) Bioenergetics 1460 (1) (2000) 133-156, https://doi.org/10.1016/S00052728(00)00135-3.

[29] E. Nango, A. Royant, M. Kubo, T. Nakane, C. Wickstrand, T. Kimura, T. Tanaka, K. Tono, C. Song, R. Tanaka, T. Arima, A. Yamashita, J. Kobayashi, T. Hosaka, E. Mizohata, P. Nogly, M. Sugahara, D. Nam, T. Nomura, T. Shimamura, D. Im, T. Fujiwara, Y. Yamanaka, B. Jeon, T. Nishizawa, K. Oda, M. Fukuda, R. Andersson, P. Båth, R. Dods, J. Davidsson, S. Matsuoka, S. Kawatake, M. Murata, O. Nureki, S. Owada, T. Kameshima, T. Hatsui, Y. Joti, G. Schertler, M. Yabashi, A.-N. Bondar, J. Standfuss, R. Neutze, S. Iwata, A three-dimensional movie of structural changes in bacteriorhodopsin, Science 354 (6319) (2016) 15521557, https://doi.org/10.1126/science.aah3497.

[30] C.D. Heyes, M.A. El-Sayed, Thermal properties of bacteriorhodopsin, J. Phys. Chem. B 107 (44) (2003) 12045-12053, https://doi.org/10.1021/ jp035327b.

[31] C.G. Brouillette, D.D. Muccio, T.K. Finney, pH dependence of bacteriorhodopsin thermal unfolding, Biochemistry 26 (23) (1987) 7431-7438, https://doi.org/ 10.1021/bi00397a035.

[32] P.-E. Milhiet, F. Gubellini, A. Berquand, P. Dosset, J.-L. Rigaud, C. Le Grimellec D. Lévy, High-resolution AFM of membrane proteins directly incorporated at high density in planar lipid bilayer, Biophys. J . 91 (9) (2006) 3268-3275, https://doi.org/10.1529/biophysj.106.087791.

[33] A. Berquand, D. Lévy, F. Gubellini, C. Le Grimellec, P.-E. Milhiet, Influence of calcium on direct incorporation of membrane proteins into in-plane lipid bilayer, Ultramicroscopy 107 (10-11) (2007) 928-933, https://doi.org/ 10.1016/j.ultramic.2007.04.008.

[34] M. Dezi, A. Di Cicco, P. Bassereau, D. Levy, Detergent-mediated incorporation of transmembrane proteins in giant unilamellar vesicles with controlled physiological contents, Proc. Nat. Acad. Sci. 110 (18) (2013) 7276-7281, https://doi.org/10.1073/pnas.1303857110.

[35] B. Andreopoulos, D. Labudde, Efficient unfolding pattern recognition in single molecule force spectroscopy data, Algorithms Mol. Biol. 6 (1) (2011) 16, https://doi.org/10.1186/1748-7188-6-16.

[36] D. Bratanov, T. Balandin, E. Round, V. Shevchenko, I. Gushchin, V. Polovinkin, V. Borshchevskiy, V. Gordeliy, An approach to heterologous expression of membrane proteins. the case of bacteriorhodopsin, PLOS ONE 10 (6) (2015) e0128390, https://doi.org/10.1371/journal.pone.0128390.

[37] V. Cherezov, J. Liu, M. Griffith, M.A. Hanson, R.C. Stevens, LCP-FRAP assay for pre-screening membrane proteins for in meso crystallization, Cryst. Growth Des. 8 (12) (2008) 4307-4315, https://doi.org/10.1021/cg800778j.

[38] T. Mukhina, V1, AFM experiments on lipid bilayers V1. doi:10.17632/ x6trfdh8zy.1.

[39] D. Necas, P. Klapetek, Gwyddion: an open-source software for SPM data analysis, Central Europe J. Phys. 10 (2012) 181-188, https://doi.org/10.2478/ s11534-011-0096-2.

[40] S.-W.W. Chen, J.-L. Pellequer, Destripe: frequency-based algorithm for removing stripe noises from AFM images, BMC Struct. Biol. 11 (2011) 7, https://doi.org/10.1186/1472-6807-11-7.

[41] S.-W.W. Chen, J.-M. Teulon, C. Godon, J.-L. Pellequer, Atomic force microscope, molecular imaging, and analysis, J. Mol. Recognit. 29 (2016) 51-55, https://doi. org/10.1002/jmr.2491.

[42] S.-W.W. Chen, A.-S. Banneville, J.-M. Teulon, J. Timmins, J.-L. Pellequer, Nanoscale surface structures of DNA bound to Deinococcus radiodurans HU unveiled by atomic force microscopy, Nanoscale 12 (2020) 22628-22638, https://doi.org/10.1039/D0NR05320A.

[43] S.-W.W. Chen, M. Odorico, M. Meillan, L. Vellutini, J.-M. Teulon, P. Parot, B. Bennetau, J.-L. Pellequer, Nanoscale structural features determined by AFM for 
single virus particles, Nanoscale 5 (2013) 10877-10886, https://doi.org/ 10.1039/C3NR02706F.

[44] C.A. Schneider, W.S. Rasband, K.W. Eliceiri, NIH image to ImageJ: 25 years of image analysis, Nature Meth. 9 (2012) 671-675, https://doi.org/10.1038/ nmeth.2089.

[45] M. Belicka, Y. Gerelli, N. Kucerka, G. Fragneto, The component group structure of DPPC bilayers obtained by specular neutron reflectometry, Soft Matter 11 (31) (2015) 6275-6283, https://doi.org/10.1039/C5SM00274E.

[46] Y. Gerelli, Aurore: new software for neutron reflectivity data analysis, J. Appl.

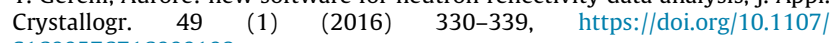
S1600576716000108.

[47] T. Crowley, E. Lee, E. Simister, R. Thomas, The use of contrast variation in the specular reflection of neutrons from interfaces, Physica B 173 (1) (1991) 143156, https://doi.org/10.1016/0921-4526(91)90044-F.

[48] J. Penfold, R.K. Thomas, The application of the specular reflection of neutrons to the study of surfaces and interfaces, J. Phys.: Condens. Matter 2 (6) (1990) 1369-1412, https://doi.org/10.1088/0953-8984/2/6/001.

[49] J. Daillant, A. Gibaud (Eds.), X-ray and Neutron Reflectivity, Lect. Notes Phys., vol. 770, Springer, Berlin Heidelberg, 2009, https://doi.org/10.1007/978-3540-88588-7.

[50] T. Saerbeck, R. Cubitt, A. Wildes, G. Manzin, K.H. Andersen, P. Gutfreund, Recent upgrades of the neutron reflectometer D17 at ILL, J. Appl. Crystallogr. 51 (2) (2018) 249-256, https://doi.org/10.1107/S160057671800239X.

[51] S. Mattauch, A. Koutsioubas, U. Rücker, D. Korolkov, V. Fracassi, J. Daemen, R. Schmitz, K. Bussmann, F. Suxdorf, M. Wagener, P. Kämmerling, H. Kleines, L. Fleischhauer-Fuß, M. Bednareck, V. Ossoviy, A. Nebel, P. Stronciwilk, S. Staringer, M. Gödel, A. Richter, H. Kusche, T. Kohnke, A. Ioffe, E. Babcock, Z. Salhi, T. Bruckel, The high-intensity reflectometer of the Jülich Centre for Neutron Science: MARIA, J. Appl. Crystallogr. 51 (3) (2018) 646-654, https:// doi.org/10.1107/S1600576718006994.

[52] Y. Gerelli, Phase transitions in a single supported phospholipid bilayer: realtime determination by neutron reflectometry, Phys. Rev. Lett. 122 (24) (2019) 248101, https://doi.org/10.1103/PhysRevLett.122.248101.

[53] J. Daillant, E. Bellet-Amalric, A. Braslau, T. Charitat, G. Fragneto, F. Graner, S. Mora, F. Rieutord, B. Stidder, Structure and fluctuations of a single floating lipid bilayer, Proc. Nat. Acad. Sci. 102 (33) (2005) 11639-11644, https://doi.org/ 10.1073/pnas.0504588102.

[54] A. Hemmerle, L. Malaquin, T. Charitat, S. Lecuyer, G. Fragneto, J. Daillant, Controlling interactions in supported bilayers from weak electrostatic repulsion to high osmotic pressure, Proc. Nat. Acad. Sci. 109 (49) (2012) 19938-19942, https://doi.org/10.1073/pnas.1211669109.

[55] J.-B. Manneville, P. Bassereau, S. Ramaswamy, J. Prost, Active membrane fluctuations studied by micropipet aspiration, Phys. Rev. E 64 (2) (2001) 021908, https://doi.org/10.1103/PhysRevE.64.021908.

[56] P. Unwin, R. Henderson, Molecular structure determination by electron microscopy of unstained crystalline specimens, J. Mol. Biol. 94 (3) (1975) 425-440, https://doi.org/10.1016/0022-2836(75)90212-0.

[57] H. Luecke, B. Schobert, H.-T. Richter, J.-P. Cartailler, J.K. Lanyi, Structure of bacteriorhodopsin at 1.55 Å resolution, J. Mol. Biol. 291 (4) (1999) 899-911, https://doi.org/10.1006/jmbi.1999.3027.

[58] N. Hasegawa, H. Jonotsuka, K. Miki, K. Takeda, X-ray structure analysis of bacteriorhodopsin at $1.3 \AA$ resolution, Sci. Rep. 8 (1) (2018) 13123, https://doi. org/10.1038/s41598-018-31370-0.

[59] G.J. Hardy, R. Nayak, S. Zauscher, Model cell membranes: Techniques to form complex biomimetic supported lipid bilayers via vesicle fusion, Curr. Opin.
Colloid Interface Sci. 18 (5) (2013) 448-458, https://doi.org/10.1016/ j.cocis.2013.06.004.

[60] J. Mou, J. Yang, Z. Shao, Atomic force microscopy of cholera toxin b-oligomers bound to bilayers of biologically relevant lipids, J. Mol. Biol. 248 (3) (1995) 507-512, https://doi.org/10.1006/jmbi.1995.0238.

[61] M.-C. Giocondi, D. Yamamoto, E. Lesniewska, P.-E. Milhiet, T. Ando, C. Le Grimellec, Surface topography of membrane domains, Biochimica et Biophysica Acta (BBA) - Biomembranes 1798 (4) (2010) 703-718, https:// doi.org/10.1016/j.bbamem.2009.09.015.

[62] Y. Jin, N. Friedman, M. Sheves, T. He, D. Cahen, Bacteriorhodopsin (bR) as an electronic conduction medium: Current transport through bR-containing monolayers 103 (23) (2006) 8601-8606. doi:10.1073/pnas.0511234103.

[63] R. Henderson, P.N.T. Unwin, Three-dimensional model of purple membrane obtained by electron microscopy, Nature 257 (5521) (1975) 28-32, https://doi. org/10.1038/257028a0.

[64] D. Fotiadis, S. Scheuring, S.A. Müller, A. Engel, D.J. Müller, Imaging and manipulation of biological structures with the AFM, Micron 33 (4)(2002)385397, https://doi.org/10.1016/S0968-4328(01)00026-9.

[65] S. Scheuring, D.J. Müller, H. Stahlberg, H.-A. Engel, A. Engel, Sampling the conformational space of membrane protein surfaces with the afm, Eur. Biophys. J. 31 (3) (2002) 172-178, https://doi.org/10.1007/s00249-001-01978.

[66] D.J. Müller, G. Büldt, A. Engel, Force-induced conformational change of bacteriorhodopsin, J. Mol. Biol. 249 (1995) 239-243, https://doi.org/10.1006/ jmbi.1995.0292.

[67] T. Soranzo, D.K. Martin, J.-L. Lenormand, E.B. Watkins, Coupling neutron reflectivity with cell-free protein synthesis to probe membrane protein structure in supported bilayers, Sci. Rep. 7 (1) 3399. doi:10.1038/s41598017-03472-8.

[68] Longpass filter, colored-glass alternative, $2 \times 2$ in., $530 \mathrm{~nm}$ cut-on (ref. 20CGA530), https://www.newport.com/p/20CGA-530.

[69] M. Andersson, E. Malmerberg, S. Westenhoff, G. Katona, M. Cammarata, A.B. Wöhri, L.C. Johansson, F. Ewald, M. Eklund, M. Wulff, J. Davidsson, R. Neutze, Structural dynamics of light-driven proton pumps, Structure 17 (9) (2009) 1265-1275, https://doi.org/10.1016/j.str.2009.07.007.

[70] J.-B. Manneville, Fluctuations de membranes actives, Ph.D. thesis, thèse de doctorat dirigée par Prost, Jacques Sciences biologiques fondamentales et appliquées, 1999.

[71] M. Shibata, H. Yamashita, T. Uchihashi, H. Kandori, T. Ando, High-speed atomic force microscopy shows dynamic molecular processes in photoactivated bacteriorhodopsin, Nat. Nanotechnol. 5 (3) (2010) 208-212, https://doi.org/ 10.1038/nnano.2010.7.

[72] A. Giahi, M. El Alaoui Faris, P. Bassereau, T. Salditt, Active membranes studied by X-ray scattering, Eur. Phys. J. E 23 (4) (2007) 431-437, https://doi.org/ 10.1140/epje/i2007-10203-7.

[73] M. Maccarini, L. Gayet, J.-P. Alcaraz, L. Liguori, B. Stidder, E.B. Watkins, J.-L. Lenormand, D.K. Martin, Functional characterization of cell-free expressed OprF porin from pseudomonas aeruginosa stably incorporated in tethered lipid bilayers, Langmuir 33 (38) (2017) 9988-9996, https://doi.org/10.1021/ acs.langmuir.7b01731.

[74] D.J. McGillivray, G. Valincius, F. Heinrich, J.W.F. Robertson, D.J. Vanderah, W. Febo-Ayala, I. Ignatjev, M. Lösche, J.J. Kasianowicz, Structure of functional staphylococcus aureus $\alpha$-hemolysin channels in tethered bilayer lipid membranes, Biophys. J. 96 (19217871) (2009) 1547-1553, https://doi.org/ 10.1016/j.bpj.2008.11.020. 